\title{
Optimal Energy-efficient Source and Relay Precoder Design for Two-way MIMO-AF Relay Systems
}

\author{
Fabien Héliot, Member, IEEE and Rahim Tafazolli, Senior Member, IEEE
}

\begin{abstract}
Energy efficiency (EE) is a key figure of merit for designing the next generation of communication systems. Meanwhile, relay-based cooperative communication, through machine-to-machine and other related technologies, is also playing an important part in the development of these systems. This paper designs an energy efficient precoding method for optimizing the EE/energy consumption of two-way multi-input multi-output (MIMO)-amplify-and-forward (AF) relay systems by using pseudo-convexity analysis to design EE-optimal precoding matrices. More precisely, we derive an EE-optimal source precoding matrix in closed-form, design a numerical approach for obtaining an optimal relay precoding matrix, prove the optimality of these matrices, when treated separately, and provide lowcomplexity bespoke algorithms to generate them. These matrices are then jointly optimized through an alternating optimization process that is proved to be systematically convergent. Performance evaluation indicates that our method can be globally optimal in some scenarios and that it is significantly more energy efficient (i.e. up to $60 \%$ more energy efficient) than existing EEbased one-way or two-way MIMO-AF precoding methods.
\end{abstract}

Index Terms-Energy efficiency, precoding/beamforming, MIMO, amplify-and-forward, two-way relay channel.

\section{INTRODUCTION}

Green and sustainable wireless communications have grown in importance over the last decade [1]-[3] to such an extent that energy efficiency (EE) is part of the design framework that has been set by the international telecommunication union (ITU) for 5G [4]. This framework targets a 100 fold improvement in $\mathrm{EE}$ going from $4 \mathrm{G}$ to $5 \mathrm{G}$. Meanwhile, the research interest for relay-based cooperative communication has also significantly grown over the last two decades [5]-[7] given that relays have been proven useful for improving the spectral efficiency (SE) [7], extending the coverage of cellular networks [7], reducing the cost of network deployments [8], or more recently increasing the EE [9], [10]. Relay-based cooperative communication is already fully integrated in wireless communication standards [11] and will undoubtedly play a significant role in machine-to-machine communications (e.g. vehicle-to-vehicle (V2V), unmanned vehicle communications) in $5 \mathrm{G}$ systems [12].

abc abc abc abc abc abc abc abc abc abc abc abc abc abc abc abc abc abc abc abc abc abc abc abc abc abc abc abc abc abc abc abc abc abc abc abc $a b c$ abc abc abc abc abc abc abc abc abc abc abc abc abc abc abc abc abc abc abc abc abc abc abc abc abc abc abc abc abc abc abc abc abc abc abc $a b c a b c a b c a b c a b c a b c a b c a b c a b c a b c a b c a b c a b c a b c a b c a b c a b c a b c$ $a b c$ abc abc abc abc abc abc abc abc abc abc abc abc abc abc abc abc abc.

The authors are with the Institute for Communication Systems, Faculty of Electronics \& Physical Sciences, University of Surrey, Guildford GU2 7XH, UK. (E-mail: f.heliot@surrey.ac.uk).

We would like to acknowledge the support of the University of Surrey 5GIC (http://www.surrey.ac.uk/5gic) for this work.
Throughout its development, numerous approaches such as amplify-and-forward (AF), decode-and-forward, or compressand-forward, have been proposed to implement relay-based communication. AF remains a popular approach due to its simplicity/practicality of implementation, its great compatibility with multi-input multi-output (MIMO) systems, and the opportunity it offers in terms of optimization (i.e. beamforming/precoding design, resource allocation) [13]. From the early work of [14], which focused on optimizing the SE of a one-way MIMO-AF system with a single relay through precoding, the design of optimal MIMO-AF precoders has been expanded to various other metrics (e.g. mean square error (MSE), transmit power, EE) and many more scenarios (e.g. multiple relays, multiple users, half/full duplex); one of these scenarios being the two-way scenario [15]-[18]. In comparison with the one-way scenario, where a relay is used to support the communication between two nodes (i.e. improves the quality/reliability of the transmission) in one direction at a time (i.e. either downlink or uplink), the communication happens in both directions at the same time (i.e. downlink and uplink) in the two-way scenario, such that the SE of twoway communication is twice as large as the SE of one-way communication in theory. When it comes to $\mathrm{EE}$, numerous MIMO-AF precoding and beamforming schemes can be found in the literature [9], [10], [19]-[23], each of them tailored for a particular scenario. For instance, joint source and relay(s) EEbased precoding optimization is considered for the one-way scenario with one relay in [9], [10], with multiple relays in [20], and for the cooperative one-way scenario with one relay in [23]. Whereas the works of [19], [21], [22] proposed EEbased beamforming (simplified version of precoding) schemes for the two-way scenario with one relay, i.e. the scenario of interest of this paper, when considering either two multiantenna nodes [19], [21] or multiple single-antenna nodes [22].

In light of these existing works, there is a clear need to design optimal precoding matrices (instead of beamforming vectors) that are specifically tailored for the two-way MIMOAF relay scenario (instead of the one-way scenario) in order to significantly improve the EE (instead of SE or MSE) of two-way MIMO-AF relay systems. This main objective has been achieved in this paper through the development of the following key contributions:

- We formulate the EE-based optimisation problem for the two-way MIMO-AF relay scenario in two different manners (one formulation being more suited for designing the precoding matrix of the source nodes, the other one being better suited for designing the relay precoding matrix), when considering a realistic power model for all 
the nodes, as it is reported in Section II. Note that the layout, achievable sum-rate, as well as power and energy consumptions of the two-way MIMO-AF relay system are also modelled in Section II.

- We derive EE-optimal precoding matrices in closed-form for the two multi-antenna source nodes of the system, based on our aforementioned optimisation problem formulation. We first reformulate the main problem for optimizing the two source precoding matrices when the relay precoder is fixed. We then prove that this reformulated problem is pseudo-convex and obtain closed-forms of the EE-optimal source precoding matrices. We finally provide a low-complexity bespoke algorithm to generate these matrices. See Section III for more details.

- We design a numerical approach for obtaining an optimal precoding matrix for a relay having multiple antennas. We first reformulate the main problem for optimizing the relay precoder when the source precoders are fixed. We then provide tight lower and upper bounds of this problem solution that are used to find the optimal one via a bespoke algorithm, which is also provided. Note that our lower bound is the solution of a pseudo-convex optimisation problem. See Section IV for more details.

- We use an alternating optimization process (e.g. [24]) to find a global solution to the main problem by combining the EE-optimal source and relay precoding matrices, but more importantly, we prove that this process is systematically convergent. We also use a convergence analysis to point out that this process can be globally optimum in some cases. See Section V for more details.

In addition, it is also worth stating that our work goes beyond the existing literature and significantly outperforms most of the relevant existing schemes for the following reasons:

- Contrary to [9], [10], [20], [23], we design optimal precoding matrices for the two-way MIMO-AF scenario instead of the one way scenario. As a result, our new design is up to $50 \%$ more energy efficient than the classic one-way transmission schemes of [9], [10] in the two-way scenario. See Section VI for more details.

- Contrary to [19], [21], [22], we design optimal precoding matrices for all the nodes in the system instead of sub-optimal beamformers/precoders (i.e. that are based on semi-definite relaxation), by considering a realistic MIMO power model. We design precoders capable of fully exploiting the MIMO channel instead of beamformers only able to exploit the best MIMO channel eigenmode. As a result, our new design is up to $60 \%$ more energy efficient than the beamforming scheme of [19] for the same two-way MIMO-AF scenario. See Section VI for more details.

- Contrary to [15]-[18], we design EE-optimal precoding matrices instead of SE-optimal or MSE-optimal matrices. In turn, our new design is up to $40 \%$ more energy efficient than the SE-based two-way MIMO-AF scheme of [16] for the same two-way MIMO-AF scenario. See Section VI for more details.

Note that a preliminary version of this work is available in

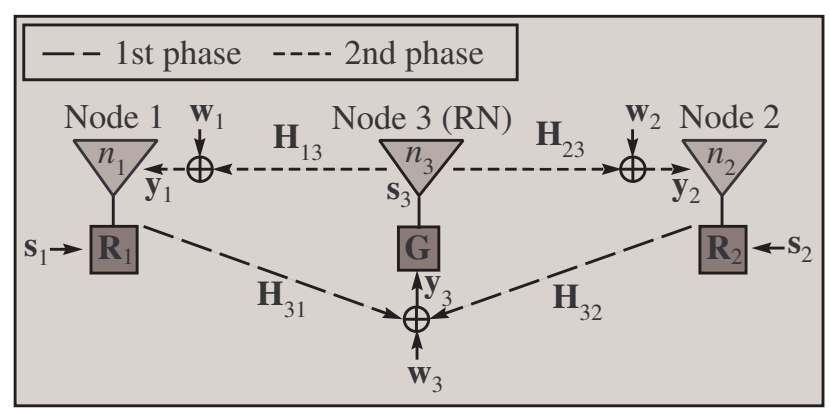

Fig. 1. Two-way MIMO-AF relay layout.

[25]; contrary to [25], we design EE-optimal precoding matrices when considering power constraints, design an improved method for obtaining the EE-optimal relay precoding matrix, and prove the convergence of our approach.

Notation: The following notation is utilized in this paper. Boldface lowercase letters (e.g. a) denote vectors, boldface uppercase letters (e.g. A) denote matrices, and boldface uppercase letters with a hat (e.g. $\widehat{\mathbf{A}}$ ) denote diagonal matrices, such that $\operatorname{diag}(\mathbf{a})=\widehat{\mathbf{A}}$, where the operator $\operatorname{diag}($.$) converts$ a vector into a diagonal matrix. Moreover, $\mathbf{I}_{x}$ denotes a $x \times x$ identity matrix, $\mathbf{0}$ denotes a zero/null matrix, $\mathbf{A} \succeq \mathbf{0}$ indicates that $\mathbf{A}$ is a positive semi-definite matrix, and $\mathbf{A}^{\frac{1}{2}}$ is the Hermitian square root of $\mathbf{A} \succeq \mathbf{0}$. Meanwhile, |.|, $\operatorname{tr}\{$.$\} ,$ .$^{\dagger}$, and.$^{-1}$ are the determinant, trace, conjugate transpose, and generalized inverse (inverse and pseudo-inverse) matrix operators, respectively. Finally, $\mathbb{C}$ denotes the set of complex number, whereas $\Leftrightarrow$ and $\Rightarrow$ are used to indicate equivalence and implication, correspondingly.

\section{TWO-WAY MIMO-AF MODEL AND EE OPTIMIZATION}

\section{A. System Layout and Model}

This paper aims at optimizing the EE/energy consumption of a half-duplex two-way MIMO-AF relay system, where three nodes having multiple antennas communicate with each other over two transmission phases, as it is depicted in Fig. 1. In the first phase, the two source nodes, i.e. Node 1 and Node 2, first use the precoding matrices $\mathbf{R}_{1} \in \mathbb{C}^{n_{1} \times n_{1}}$ and $\mathbf{R}_{2} \in \mathbb{C}^{n_{2} \times n_{2}}$ to precode their transmit signals $\mathbf{s}_{1} \in \mathbb{C}^{n_{1} \times 1}$ and $\mathbf{s}_{2} \in \mathbb{C}^{n_{2} \times 1}$, respectively, with $n_{i}, i=1,2$, being the number of antennas at Node $i$. Note also that $E\left\{\mathbf{s}_{i} \mathbf{s}_{i}^{\dagger}\right\}=\mathbf{I}_{n_{i}}, i=1,2$, where $E\{$.$\} is$ the expectation operator. These two precoded signals are then sent to Node 3, a.k.a the relay node (RN), over the MIMO channels $\mathbf{H}_{31} \in \mathbb{C}^{n_{3} \times n_{1}}$ and $\mathbf{H}_{32} \in \mathbb{C}^{n_{3} \times n_{2}}$, correspondingly, such that the received signal at the $\mathrm{RN}$ can be expressed as

$$
\mathbf{y}_{3}=\sum_{i=1}^{2} \mathbf{H}_{3 i} \mathbf{R}_{i} \mathbf{s}_{i}+\mathbf{w}_{3},
$$

where $\mathbf{w}_{3} \in \mathbb{C}^{n_{3} \times 1}$ models the noise at the $\mathrm{RN}$ and $n_{3}$ is the number of antennas at the RN. Note that in here, as in [15]-[18], the direct link communication between Node 1 and Node 2 is assumed to be unreliable, which justifies the need for a RN. In the second phase, the RN broadcasts the amplified signal $\mathbf{s}_{3}=\mathbf{G y}_{3}$, where $\mathbf{G} \in \mathbb{C}^{n_{3} \times n_{3}}$ is the $\mathrm{RN}$ precoding 
matrix, to both Node 1 and Node 2 over the MIMO channels $\mathbf{H}_{13} \in \mathbb{C}^{n_{1} \times n_{3}}$ and $\mathbf{H}_{23} \in \mathbb{C}^{n_{2} \times n_{3}}$, correspondingly, such that the received signal at Node $i$ can be expressed as

$$
\mathbf{y}_{i}=\mathbf{H}_{i 3} \mathbf{G H}_{3 i} \mathbf{R}_{i} \mathbf{s}_{i}+\mathbf{H}_{i 3} \mathbf{G H}_{3 \bar{i}} \mathbf{R}_{\bar{i}} \mathbf{s}_{\bar{i}}+\mathbf{H}_{i 3} \mathbf{G w}_{3}+\mathbf{w}_{i},
$$

where $\mathbf{w}_{i} \in \mathbb{C}^{n_{i} \times 1}, i=1,2$, models the noise at Node $i$ and $\bar{i} \triangleq 3-i$. It can clearly be seen that $\mathbf{H}_{i 3} \mathbf{G H}_{3 i} \mathbf{R}_{i} \mathbf{s}_{i}$ in (2) is a self-interference term, given that $\mathbf{s}_{i}$ is the transmit signal of Node $i$. Assuming as in [15]-[18] that full channel state information (CSI) is available at all the nodes, then this selfinterference can be fully removed and the aggregate mutual information/achievable rate (over the two transmission phases) of the two-way MIMO-AF system in Fig. 1 can be formulated, in two manners, as $R_{\Sigma}(\mathbf{R}, \mathbf{G})=$

$$
\begin{aligned}
W \sum_{i=1}^{2} \log _{2}\left|\mathbf{I}_{n_{\bar{i}}}+\frac{\sigma_{3}^{2} \mathbf{H}_{\bar{i} 3} \mathbf{G} \dot{\mathbf{\Upsilon}}_{i}(\mathbf{R}) \mathbf{G}^{\dagger} \mathbf{H}_{\bar{i} 3}^{\dagger}}{\sigma_{\bar{i}}^{2} \mathbf{I}_{n_{\bar{i}}}+\sigma_{3}^{2} \mathbf{H}_{\bar{i} 3} \mathbf{G} \mathbf{G}^{\dagger} \mathbf{H}_{\bar{i} 3}^{\dagger}}\right| \text { or } \\
W \log _{2}\left|\mathbf{I}_{n_{0}}+\mathbf{R}^{\dagger} \dot{\mathbf{\Psi}}(\mathbf{G}) \mathbf{R}\right| .
\end{aligned}
$$

Further practical details about CSI acquisition can be found in [13], [26]. Equation (3a) is equivalent to the sum-rate formulation of [16] over two phases; whereas (3b) is a reformulation of (3a) based on the matrix determinant lemma and the property of block matrix determinant, for which more details are provided in Section A of the Appendix. It can be remarked in (3a) that the aggregate rate of the two-way MIMO-AF system is equivalent to the sum of the rates of two one-way MIMO-AF systems (see equation (15) of [14]). In (3), $W$ is the channel bandwidth, $n_{0}=n_{1}+n_{2}$, and $\mathbf{R}=\left[\mathbf{R}_{1} \mathbf{0} ; \mathbf{0} \mathbf{R}_{2}\right]$ as well as $\dot{\Psi}(\mathbf{G})=\left[\dot{\Psi}_{1}(\mathbf{G}) \mathbf{0} ; \mathbf{0} \dot{\Psi}_{2}(\mathbf{G})\right]$ are block diagonal matrices in $\mathbb{C}^{n_{0} \times n_{0}}$. In addition,

$$
\begin{aligned}
& \dot{\boldsymbol{\Upsilon}}_{i}(\mathbf{R})=\sigma_{3}^{-2} \mathbf{H}_{3 i} \mathbf{R}_{i} \mathbf{R}_{i}^{\dagger} \mathbf{H}_{3 i}^{\dagger}, \\
& \dot{\mathbf{\Psi}}_{i}(\mathbf{G})=\mathbf{H}_{3 i}^{\dagger} \mathbf{G}^{\dagger} \mathbf{H}_{\bar{i} 3}^{\dagger}\left(\sigma_{\bar{i}}^{2} \mathbf{I}_{n_{\bar{i}}}+\sigma_{3}^{2} \mathbf{H}_{\bar{i} 3} \mathbf{G} \mathbf{G}^{\dagger} \mathbf{H}_{\bar{i} 3}^{\dagger}\right)^{-1} \mathbf{H}_{\bar{i} 3} \mathbf{G} \mathbf{H}_{3 i},
\end{aligned}
$$

$i=1,2$, and $\sigma_{j}^{2}$ models the variance of the Gaussian noise at Node $j, j=1,2,3$.

\section{B. Power Consumption Model}

The power consumption of common communication nodes, e.g. base station (BS), RN or user equipment (UE), is proportional to their transmit power according to [27]-[29]. Hence, in a MIMO context, it is usually formulated as [30], [31]

$$
\begin{aligned}
P_{j}^{\mathrm{Tx}} & =\Delta_{j} P_{j}+n_{j} P_{j}^{\mathrm{CipA}}+P_{j}^{\mathrm{Ci}} \text { or } \\
P_{j}^{\mathrm{Rx}} & =\varsigma\left[n_{j} P_{j}^{\mathrm{CipA}}+P_{j}^{\mathrm{Ci}}\right],
\end{aligned}
$$

when the $j$-th node is transmitting or receiving, respectively, $j=1,2,3$. In (4), $P_{j}$ represents the transmit power, $\Delta_{j}$ models the power amplifier inefficiency, $P_{j}^{\text {CipA }}$ is the perantenna circuit power consumption (e.g. radio frequency (RF) transceiver chain), and $P_{j}^{\mathrm{Ci}}$ represents other circuit power consumptions (e.g. baseband processing) at node $j$. In addition, $0 \leq \varsigma \leq 1$, since receiving consumes generally less circuit power than transmitting. Hence, based on the communication layout of Fig. 1 and the power consumption equations in (4), the total consumed power (over the two transmission phases) of a two-way MIMO-AF system, can be expressed as

$$
\begin{aligned}
P_{\Sigma} & =\left(\sum_{i=1}^{2} P_{i}^{\mathrm{Tx}}+P_{3}^{\mathrm{Rx}}\right)+\left(P_{3}^{\mathrm{Tx}}+\sum_{i=1}^{2} P_{i}^{\mathrm{Rx}}\right) \\
& =P_{c}+\sum_{i=1}^{3} \Delta_{i} P_{i} .
\end{aligned}
$$

Equation (5a) reflects the two-phases transmission of the data, i.e. Nodes 1 and 2 transmit data that are received by the $\mathrm{RN}$ in the first phase; whereas the $\mathrm{RN}$ transmits data that are received by Nodes 1 and 2 in the second phase. Meanwhile, equation (5b) is obtained by inserting (4) into (5a), such that

$$
P_{c}=(1+\varsigma)\left(\sum_{i=1}^{3} n_{i} P_{i}^{\mathrm{CipA}}+P_{i}^{\mathrm{Ci}}\right) .
$$

It can be remarked in (5a) that contrary to one-way MIMOAF [10], [20] or, more generally, cooperative MIMO-AF [23], none of the three nodes are idle in the two-way scenario, which is clearly more power efficient. Given that, based on [16],

$$
\begin{aligned}
P_{i}(\mathbf{R}) & =\operatorname{tr}\left\{\mathbf{R}_{i} \mathbf{R}_{i}^{\dagger}\right\}, i=1,2, \text { and } \\
P_{3}(\mathbf{R}, \mathbf{G}) & =\operatorname{tr}\left\{\mathbf{G}\left(\sigma_{3}^{2} \mathbf{I}_{n_{3}}+\sum_{i=1}^{2} \mathbf{H}_{3 i} \mathbf{R}_{i} \mathbf{R}_{i}^{\dagger} \mathbf{H}_{3 i}^{\dagger}\right) \mathbf{G}^{\dagger}\right\},
\end{aligned}
$$

$P_{\Sigma}$ can then be re-formulated, by inserting (7) into (5b), as

$$
P_{\Sigma}(\mathbf{R}, \mathbf{G})=P_{c}+\sum_{i=1}^{2} \Delta_{i} P_{i}(\mathbf{R})+\Delta_{3} P_{3}(\mathbf{R}, \mathbf{G}) .
$$

\section{Energy Consumption and Optimization}

The energy consumption, $E_{b}$ (in J/bit), or EE (in bit/J), is a ratio between $P_{\Sigma}$ and $R_{\Sigma}$ [32], such that the energy consumption of Figure 1's system can be modeled as

$$
E_{b}(\mathbf{R}, \mathbf{G})=\frac{P_{\Sigma}(\mathbf{R}, \mathbf{G})}{R_{\Sigma}(\mathbf{R}, \mathbf{G})}
$$

where $R_{\Sigma}(\mathbf{R}, \mathbf{G})$ and $P_{\Sigma}(\mathbf{R}, \mathbf{G})$ are expressed in (3) and (8), respectively. The main aim of this paper being to minimize this energy consumption, we focus on solving (in the subsequent sections) the following optimization problem

$$
\begin{aligned}
\min _{\mathbf{R}, \mathbf{G}} & E_{b}(\mathbf{R}, \mathbf{G}), \\
\text { s.t. } & P_{i}(\mathbf{R}) \leq P_{i}^{\max }, i=1,2, \\
& P_{3}(\mathbf{R}, \mathbf{G}) \leq P_{3}^{\max } .
\end{aligned}
$$

In other words, our aim is to find precoding matrices $\mathbf{R}$ and $\mathbf{G}$ minimizing the energy consumption of the two-way MIMOAF system in Fig. 1, when assuming that the transmit power of any of the three nodes is constrained. Note that $P_{j}^{\max }$ in (10) is the maximum transmit power of the $j$-th node, $j=1,2,3$.

The objective function in (10a) is not necessarily jointly convex in $\mathbf{R}_{1}, \mathbf{R}_{2}$, and $\mathbf{G}$, which makes the problem in (10) generally non-convex and, hence, not straightforward to solve. However, it becomes possible to efficiently solve this problem when $\mathbf{R}=\left[\mathbf{R}_{1} \mathbf{0} ; \mathbf{0} \mathbf{R}_{2}\right]$ and $\mathbf{G}$ are treated independently. For instance, we first prove in Section III 
that the optimization problem in (10) can be expressed in a pseudo-convex form when $\mathbf{G}$ is known/fixed; we then derive an optimal matrix $\mathbf{R}^{\star}=\left[\mathbf{R}_{1}^{\star} \mathbf{0} ; \mathbf{0} \mathbf{R}_{2}^{\star}\right]$, in closed-form, to minimize (10). Similarly, we design in Section IV a smart method for deriving an optimal matrix $\mathbf{G}^{\star}$ to minimize (10) when $\mathbf{R}$ is known. Then, an alternating optimization process is used in Section V to jointly optimize the outcomes of Section III and IV and find a global solution for (10).

\section{EE-Optimal Source Precoder $\mathbf{R}^{\star}$}

\section{A. Problem Reformulation and closed-form of $\mathbf{R}^{\star}$}

Finding the EE-optimal source precoding matrix, $\mathbf{R}^{\star}$, when $\mathbf{G}$ is known (i.e. $\mathbf{G}$ is not a variable in this subsection and, hence, is omitted from most of the equations), can be done by solving the optimization problem in (10) with $E_{b}(\mathbf{R}, \mathbf{G})$ as in (9), and where $R_{\Sigma}(\mathbf{R}, \mathbf{G})$ and $P_{\Sigma}(\mathbf{R}, \mathbf{G})$ are given in (3b) and (8), respectively.

Proposition 1: This problem can be reformulated in an equivalent form as

$$
\begin{aligned}
& \min _{\mathbf{Y}} E_{b}(\mathbf{Y})=\frac{P_{c}+\sum_{i=1}^{3} \Delta_{i} P_{i}(\mathbf{Y})}{R_{\Sigma}(\mathbf{Y})}, \\
& \text { s.t. } P_{i}(\mathbf{Y}) \leq P_{i}^{\max }, i=1,2,3,
\end{aligned}
$$

where

$$
\begin{aligned}
R_{\Sigma}(\mathbf{Y}) & =W \log _{2}\left|\mathbf{I}_{n_{0}}+\mathbf{Y}\right|, \\
P_{i}(\mathbf{Y}) & =\operatorname{tr}\left\{\mathbf{Y} \mathbf{\Psi}_{i}\right\}+[i-2]_{+} \sigma_{3}^{2} \operatorname{tr}\left\{\mathbf{G G}^{\dagger}\right\},
\end{aligned}
$$

$i=1,2,3,[\cdot]_{+}$refers to $\max \{., 0\}$ and $\mathbf{Y}$ is a block diagonal matrix. In addition,

$$
\begin{aligned}
& \boldsymbol{\Psi}_{1}=\left[\begin{array}{cc}
\dot{\boldsymbol{\Psi}}_{1}^{-1} & \mathbf{0} \\
\mathbf{0} & \mathbf{0}
\end{array}\right], \boldsymbol{\Psi}_{2}=\left[\begin{array}{cc}
\mathbf{0} & \mathbf{0} \\
\mathbf{0} & \dot{\boldsymbol{\Psi}}_{2}^{-1}
\end{array}\right], \text { and } \\
& \boldsymbol{\Psi}_{3}=\left[\begin{array}{ccc}
\dot{\boldsymbol{\Psi}}_{1}^{-\frac{1}{2}} \mathbf{H}_{31}^{\dagger} \mathbf{G}^{\dagger} \mathbf{G} \mathbf{H}_{31} \dot{\boldsymbol{\Psi}}_{1}^{-\frac{1}{2}} & \mathbf{0} \\
\mathbf{0} & \dot{\boldsymbol{\Psi}}_{2}^{-\frac{1}{2}} \mathbf{H}_{32}^{\dagger} \mathbf{G}^{\dagger} \mathbf{G} \mathbf{H}_{32} \dot{\boldsymbol{\Psi}}_{2}^{-\frac{1}{2}}
\end{array}\right]
\end{aligned}
$$

Proof: By applying the change of variable

$$
\mathbf{Y}=\dot{\boldsymbol{\Psi}}^{\frac{1}{2}} \mathbf{R} \mathbf{R}^{\dagger} \dot{\boldsymbol{\Psi}}^{\frac{1}{2}} \succeq \mathbf{0}, \mathbf{Y} \in \mathbb{C}^{n_{0} \times n_{0}},
$$

to equation (3b), the latter can simply be re-expressed as (12a). In addition, it can for instance be easily shown that $P_{1}(\mathbf{Y})=\operatorname{tr}\left\{\mathbf{Y} \boldsymbol{\Psi}_{1}\right\}=\operatorname{tr}\left\{\dot{\boldsymbol{\Psi}}^{\frac{1}{2}} \mathbf{R} \mathbf{R}^{\dagger} \dot{\boldsymbol{\Psi}}^{\frac{1}{2}}\left[\dot{\mathbf{\Psi}}_{1}^{-1} \mathbf{0} ; \mathbf{0} \mathbf{0}\right]\right\}=$ $\operatorname{tr}\left\{\dot{\boldsymbol{\Psi}}^{\frac{1}{2}} \mathbf{R} \mathbf{R}^{\dagger} \dot{\boldsymbol{\Psi}}^{\frac{1}{2}} \dot{\boldsymbol{\Psi}}^{-\frac{1}{2}}\left[\mathbf{I}_{n_{1}} \mathbf{0} ; \mathbf{0} \mathbf{0}\right] \dot{\boldsymbol{\Psi}}^{-\frac{1}{2}}\right\}=\operatorname{tr}\left\{\left[\mathbf{R}_{1} \mathbf{0} ; \mathbf{0} \mathbf{R}_{2}\right]^{\dagger}\right.$ $\left.\times\left[\mathbf{I}_{n_{1}} \mathbf{0} ; \mathbf{0} 0 \mathbf{0}\right]\left[\mathbf{R}_{1} \mathbf{0} ; \mathbf{0} \mathbf{R}_{2}\right]\right\}=\operatorname{tr}\left\{\mathbf{R}_{1}^{\dagger} \mathbf{R}_{1}\right\}=\operatorname{tr}\left\{\mathbf{R}_{1} \mathbf{R}_{1}^{\dagger}\right\}=$ $P_{1}(\mathbf{R})$, according to (13) and (14). Note that the equivalence between $P_{2}(\mathbf{Y})$ and $P_{2}(\mathbf{R})$ as well as $P_{3}(\mathbf{Y})$ and $P_{3}(\mathbf{R})$ can be proved in the same manner.

Proposition 2: The optimization problem in (11) is a pseudo-convex problem, where its objective function in (11a) is strictly pseudo-convex and its constraint functions in (11b) are linear (hence, convex and pseudo-convex) functions of $\mathbf{Y}$.

Proof: the function $E_{b}$ in (11a) is a function of the type $p / q$, where $p$ is a linear function and $q$ is a strictly concave function. According to [23], such functions are strictly pseudoconvex.

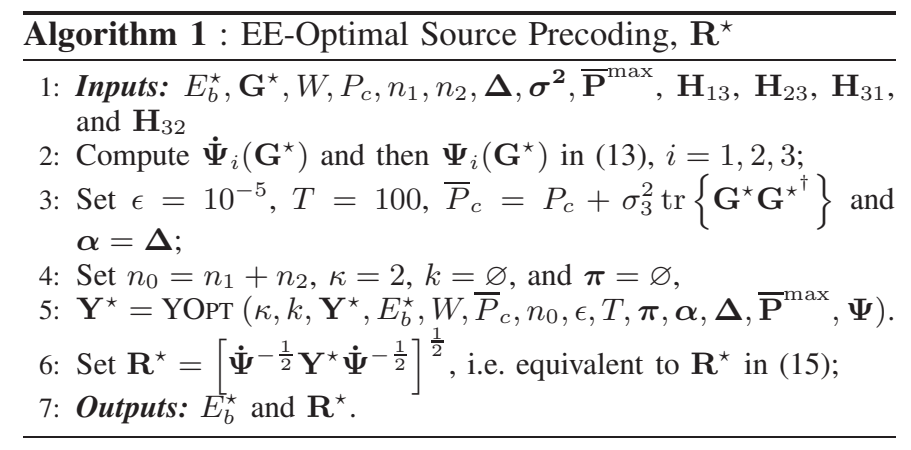

Proposition 3: The EE-optimal source precoding matrix, $\mathbf{R}^{\star}$, i.e. the optimal argument of (10) when $\mathbf{G}$ is fixed, can be formulated in closed-form as

$$
\mathbf{R}^{\star}=\left[\dot{\Psi}^{-\frac{1}{2}}\left(\frac{W E_{b}^{\star}}{\ln (2)}\left(\sum_{i=1}^{3} \alpha_{i} \boldsymbol{\Psi}_{i}\right)^{-1}-\mathbf{I}_{n_{0}}\right) \dot{\Psi}^{-\frac{1}{2}}\right]^{\frac{1}{2}},
$$

where $E_{b}^{\star}=E_{b}\left(\mathbf{R}^{\star}, \mathbf{G}\right)$ is the optimal solution of (11), i.e. the optimal solution of (10) when $\mathbf{G}$ is fixed. In addition, $\boldsymbol{\alpha}=\left[\alpha_{1}, \alpha_{2}, \alpha_{3}\right] \in \mathbb{R}_{\geq 0}^{3}$, where $\alpha_{i}=\delta_{i}^{\star} \Delta_{i}+\left(1-\delta_{i}^{\star}\right) \mu_{i}^{\star}$, $\mu_{i}^{\star} \geq 0$ is a Lagrangian multiplier, and $\delta^{\star}=\left[\delta_{1}^{\star}, \delta_{2}^{\star}, \delta_{3}^{\star}\right]$ with

$$
\delta_{i}^{\star}= \begin{cases}1, & \text { if } P_{i}\left(\mathbf{R}^{\star}\right) \leq P_{i}^{\max } ; \\ 0, & \text { else. }\end{cases}
$$

Proof: See section B of the Appendix.

\section{B. Solving (11) and finding $\mathbf{R}^{\star}$}

Given that the optimization problem in (11) is pseudoconvex, where its objective function is a ratio of a linear to a concave function, it can be solved by using a generic fractional optimization method as, for instance, the method proposed in [33]. However, it is possible to design a bespoke and, hence, more efficient low-complexity algorithm for solving (11) by leveraging on the closed-form of $\mathbf{R}^{\star}$ in (15).

The objective function in (11a) is continuous, but not constant, over its domain and it goes towards infinity at both the extrema of its domain (i.e. when $\mathbf{Y}$ goes towards zero or infinity), as long as $P_{c}>0$. Thus, (11a) has a minimum, which is not infinity. Moreover, we know from optimization theory [34] that adding constraints to a problem either maintains or reduces the domain of the problem and, hence, either maintains or reduces its set of possible/feasible solutions. In other words, the minimum of (11a) is always lower or equal to the optimal solution of the whole problem in (11). Consequently, it is possible to effectively solve (11) and optimally obtain $\mathbf{R}^{\star}$ as well as $E_{b}^{\star}=E_{b}\left(\mathbf{R}^{\star}, \mathbf{G}\right)$ by following the subsequent steps, which are fully detailed in Algorithm 1 and its dependencies, i.e. "YOpт" and "PowCstOpt": first, by obtaining the global minimum of (11a) when assuming no constraints (i.e. without (11b)); second, by refining the value of this minimum if at least one of the constraints in (11b) is not met when this minimum is reached. The functions "YOPT" and "PowCstOpt", which are subsequently provided on the next page, are recursive functions that are based on standard univariate root-finding algorithms. For instance, "РоwCsтOрт" 


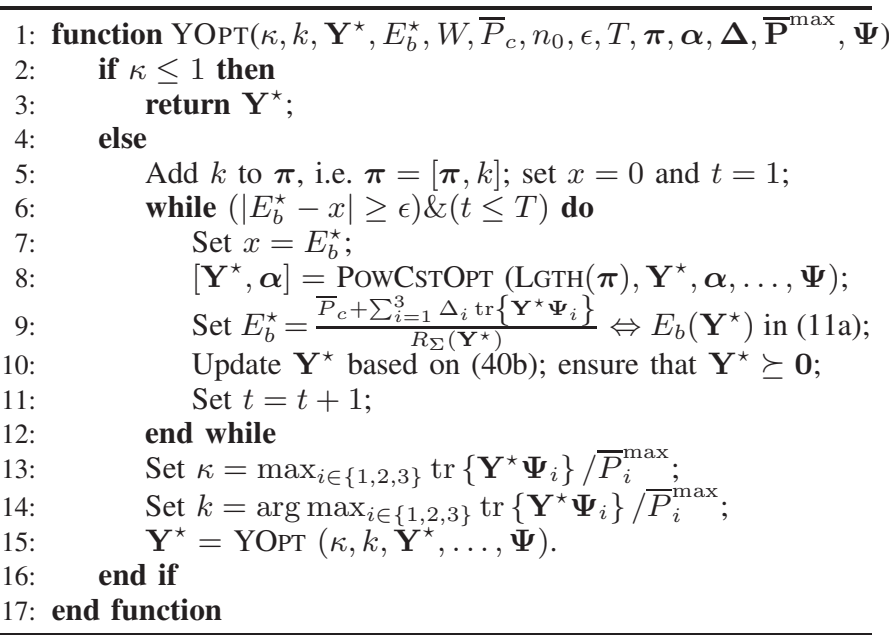

relies on the Newton-Raphson method [35], as it is indicated in line 6 of the function "PowCstOpT", which is typically used for solving water-filling problems. The function "YOPT" computes the optimum argument of $E_{b}(\mathbf{Y})$ in (11a), i.e. $\mathbf{Y}^{\star}$, whereas "PowCstOpt" refines the value of $\mathbf{Y}^{\star}$ for all the possible power constraint cases, which are detailed thereafter. Note that in "YOpт", "РоwCstOpT", and Algorithm 1, $\varnothing$ represents an empty vector, $\epsilon$ sets the accuracy of the algorithms, $T$ is the maximum number of iterations of the algorithms, $\pi$ is a vector of indices, $\overline{\mathbf{P}}^{\max }=\left[P_{1}^{\max }, P_{2}^{\max }, P_{3}^{\max }-\sigma_{3}^{2} \operatorname{tr}\left\{\mathbf{G G}^{\dagger}\right\}\right]$, $\boldsymbol{\sigma}^{\mathbf{2}}=\left[\sigma_{1}^{2}, \sigma_{2}^{2}, \sigma_{3}^{2}\right], \mathbf{\Psi}=\left[\boldsymbol{\Psi}_{1}, \boldsymbol{\Psi}_{2}, \boldsymbol{\Psi}_{3}\right]$, and the function "LGTH" returns the length of a vector.

1) Unconstrained Optimization: Finding the global minimum of (11a), i.e. solving (11) in the unconstrained case, is equivalent to solving a single equation, i.e. $E_{b}^{\star}=E_{b}(\mathbf{Y}=$ $\left.\mathbf{Y}^{\star}\right)$, with one unknown, i.e. $E_{b}^{\star}$. Indeed, in the case that the power constraints are not enforced, then $\boldsymbol{\delta}^{\star}=[1,1,1]$; in turn, it implies that $\boldsymbol{\alpha}=\boldsymbol{\Delta}=\left[\Delta_{1}, \Delta_{2}, \Delta_{3}\right]$, such that $\mathbf{Y}^{\star}$ in (40b) or $\mathbf{R}^{\star}$ in (15) is only dependent of the variable $E_{b}^{\star}$.

2) Power Constraint Optimization: In the case that $\delta_{i}^{\star}=0$ for at least $i=1,2$, or 3 , then the problem in (11) becomes in effect power constrained, i.e. equivalent to a water-filling problem. Given that $\delta^{\star}$ contains three elements that are either 0 or 1 , and that $\boldsymbol{\delta}^{\star}=[1,1,1]$ represents the unconstrained case, there are seven different power constrained cases. They can be categorized in three groups: single, dual, or full power constrained cases.

In the single power constrained case, i.e. if $\boldsymbol{\delta}^{\star}=[0,1,1]$, $[1,0,1]$, or $[1,1,0]$, only one (out of the three) power constraint in $(11 \mathrm{~b})$ is active, i.e. $P_{i}\left(\mathbf{Y}^{\star}\right)=P_{i}^{\max }$, for either $i=1,2$ or 3 . Consequently, finding the global minimum of (11) is equivalent to solving a system of two equations, i.e. $E_{b}^{\star}=E_{b}\left(\mathbf{Y}=\mathbf{Y}^{\star}\right)$ and $P_{i}\left(\mathbf{Y}^{\star}\right)=P_{i}^{\max }$, with two unknowns, i.e. $E_{b}^{\star}$ and $\mu_{i}^{\star}$. For instance, if $\boldsymbol{\delta}^{\star}=[1,0,1]$, then $\boldsymbol{\alpha}=\left[\Delta_{1}, \mu_{2}^{\star}, \Delta_{3}\right]$, such that $\mathbf{Y}^{\star}$ in (40b) is dependent of both $E_{b}^{\star}$ and $\mu_{2}^{\star}$.

In the dual power constrained case, i.e. if $\boldsymbol{\delta}^{\star}=[0,0,1]$, $[0,1,0]$, or $[1,0,0]$, two (out of the three) power constraints in (11b) are active. Consequently, finding the global minimum of (11) is equivalent to solving a system of three equations, i.e. $E_{b}^{\star}=E_{b}\left(\mathbf{Y}=\mathbf{Y}^{\star}\right)$ and two $P_{i}\left(\mathbf{Y}^{\star}\right)=P_{i}^{\max }$ equations,

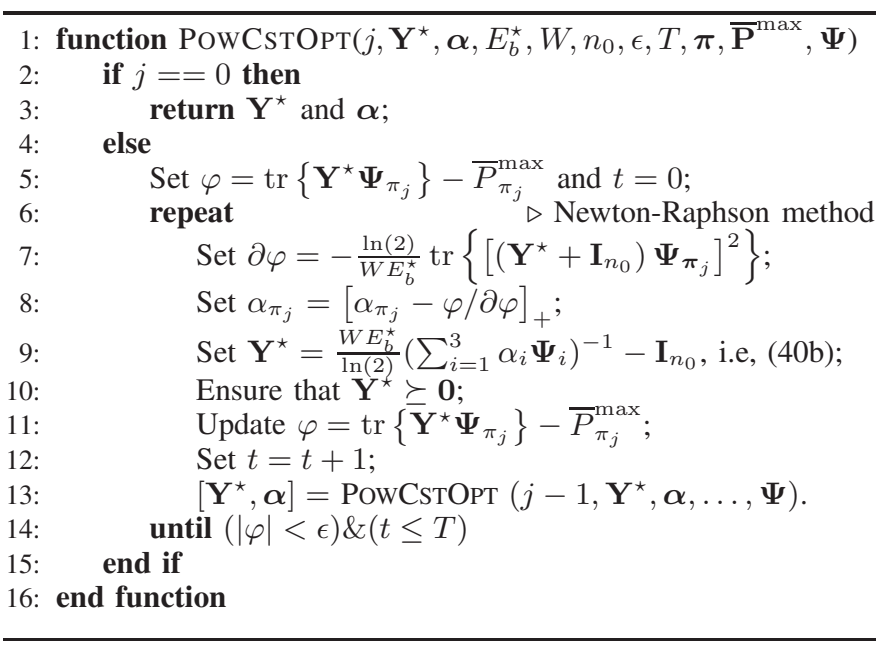

with three unknowns, i.e. $E_{b}^{\star}$ and two values of $\mu_{i}^{\star}$.

In the full power constrained case, i.e. if $\delta^{\star}=[0,0,0]$, all the power constraints in (11b) are active and, hence, the numerator of (11a) becomes a constant. In turn, the problem in (11) becomes a rate optimization problem, i.e. minimizing the inverse of a sum-rate subject to three power constraints. In this case, finding the global minimum of (11) is also equivalent to solving a system of three equations, i.e. $P_{i}\left(\mathbf{Y}^{\star}\right)=P_{i}^{\max }$ with three unknowns, i.e. $\mu_{i}^{\star}, i=1,2,3$, where $E_{b}^{\star}$ acts as a proportionality constant.

Finally, it is important to note that the problem in (11) and, hence Algorithm 1, has always a solution as long as $\bar{P}_{i}^{\max } \geq$ $0, \forall i=1,2,3$. Thus, it is important to check that $P_{3}^{\max }-$ $\sigma_{3}^{2} \operatorname{tr}\left\{\mathbf{G G}^{\dagger}\right\} \geq 0$, based on (12b), prior to run Algorithm 1 .

\section{EE-Optimal Relay Precoder $\mathbf{G}^{\star}$}

Finding the EE-optimal relay precoding matrix, $\mathbf{G}^{\star}$, when $\mathbf{R}$ is known (i.e. $\mathbf{R}$ is not a variable here and is omitted from most of the equations), can be done by solving the optimization problem in (10) with $E_{b}(\mathbf{R}, \mathbf{G})$ as in (9), and where $R_{\Sigma}(\mathbf{R}, \mathbf{G})$ and $P_{\Sigma}(\mathbf{R}, \mathbf{G})$ are given in (3a) and (8), respectively. This problem can be reformulated as

$$
\begin{aligned}
& \min _{\mathbf{G}} E_{b}(\mathbf{G})=\frac{P_{c}^{\prime}+\Delta_{3} P_{3}(\mathbf{G})}{R_{\Sigma}(\mathbf{G})}, \\
& \text { s.t. } P_{3}(\mathbf{G}) \leq P_{3}^{\max },
\end{aligned}
$$

where $P_{c}^{\prime}=P_{c}+\sum_{i=1}^{2} \Delta_{i} P_{i}$ and $P_{3}(\mathbf{G})$ is defined in $(7 \mathrm{~b})$. Contrary to (11), it is not feasible to express (17) in a pseudoconvex form and, hence, to solve (17) in a straightforward manner. Instead, we propose in the following to first obtain lower and upper bounds of the optimal solution of (17), i.e. $E_{b}^{\star}=E_{b}\left(\mathbf{R}, \mathbf{G}^{\star}\right)$, and then to use a simple method (e.g. bisection method) to find $E_{b}^{\star}$ based on these bounds.

\section{A. Lower bound to the optimal solution of (17)}

In order to find a lower bound, $E_{b}^{\mathbf{\nabla}}$, to the optimal solution of (17), i.e. $E_{b}^{\boldsymbol{\nabla}} \leq E_{b}^{\star}$, we first reformulate (17) and relax its formulation by omitting one constraint. We then explain how to solve its relaxed form in a low-complexity and optimal manner for obtaining $E_{b}^{\mathbf{V}}$. 
Proposition 4: Let $\overline{\mathbf{G}} \in \mathbb{C}^{2 n_{3} \times 2 n_{3}}$ be a block diagonal matrix defined as

$$
\overline{\mathbf{G}}=\left[\begin{array}{lc}
\mathbf{G}_{1} & \mathbf{0} \\
\mathbf{0} & \mathbf{G}_{2}
\end{array}\right],
$$

then the optimisation problem in (17) can be reformulated as

$$
\begin{array}{ll}
\min _{\overline{\mathbf{G}}} & E_{b}(\overline{\mathbf{G}})=\frac{P_{c}^{\prime}+\Delta_{3} P_{3}(\overline{\mathbf{G}})}{R_{\Sigma}(\overline{\mathbf{G}})}, \\
\text { s.t. } & P_{3}(\overline{\mathbf{G}}) \leq P_{3}^{\max }, \\
& \mathbf{G}_{1}=\mathbf{G}_{2}=\mathbf{G} \text { in (19), }
\end{array}
$$

where $R_{\Sigma}$ in (3a) and $P_{3}$ in (7b) can respectively be reformulated as

$$
R_{\Sigma}(\overline{\mathbf{G}})=W \log _{2}\left|\mathbf{I}_{n_{0}}+\mathbf{H}_{3} \overline{\mathbf{G}} \Upsilon \overline{\mathbf{G}}^{\dagger} \mathbf{H}_{3}^{\dagger}\left(\mathbf{I}_{n_{0}}+\mathbf{H}_{3} \overline{\mathbf{G G}}^{\dagger} \mathbf{H}_{3}^{\dagger}\right)^{-1}\right|,
$$

$$
P_{3}(\overline{\mathbf{G}})=\sigma_{3}^{2} \operatorname{tr}\left\{\overline{\mathbf{G}}\left(\frac{\mathbf{I}_{2 n_{3}}}{2}+\mathbf{\Upsilon}\right) \overline{\mathbf{G}}^{\dagger}\right\} .
$$

In addition,

$$
\mathbf{H}_{3}=\frac{\sigma_{3}}{\sigma_{1} \sigma_{2}}\left[\begin{array}{cc}
\sigma_{1} \mathbf{H}_{23} & \mathbf{0} \\
\mathbf{0} & \sigma_{2} \mathbf{H}_{13}
\end{array}\right] \text { and } \boldsymbol{\Upsilon}=\left[\begin{array}{cc}
\dot{\boldsymbol{\Upsilon}}_{1} & \mathbf{0} \\
\mathbf{0} & \dot{\boldsymbol{\Upsilon}}_{2}
\end{array}\right] \text {. }
$$

Proof: See section C of the Appendix.

Proposition 5: The optimal solution of the relaxed form of the optimisation problem in (19), i.e. when the constraint (19c) (enforcing that the upper, $\mathbf{G}_{1}$, and lower, $\mathbf{G}_{2}$, parts of $\overline{\mathbf{G}}$ are the same) is omitted, is a lower bound to the optimal solution of both problems (17) and (19), which are equivalent.

Proof: As it has been previously mentioned in the second paragraph of Section III-B, we know from optimization theory [34] that adding constraints to an optimization problem either maintains or reduces the set of its possible/feasible solutions such that the optimal solution of a minimization problem with added constraints can only be larger or equal to the optimal solution of its original problem. In other words, the minimum value of the problem with added constraints upper bounds the minimum value of its original problem.

It can be remarked that the relaxed form of (19) (i.e. when constraint (19c) is omitted) is similar to finding the optimum relay matrix $\overline{\mathbf{G}}$ of a classic one-way MIMO-AF system with a single relay [9], [14]. Accordingly, the optimum relay precoder matrix for the relaxed version of (19) is of the form

$$
\overline{\mathbf{G}}=\mathbf{V} \widehat{\mathbf{G}}^{\frac{1}{2}} \mathbf{U}^{\dagger},
$$

where $\mathbf{V}=\left[\begin{array}{cc}\mathbf{V}_{1} & \mathbf{0} \\ \mathbf{0} & \mathbf{V}_{2}\end{array}\right]$ as well as $\mathbf{U}=\left[\begin{array}{cc}\mathbf{U}_{1} & \mathbf{0} \\ \mathbf{0} & \mathbf{U}_{2}\end{array}\right]$ are unitary block diagonal matrices that contains the right and leftsingular vectors of $\mathbf{H}_{3}$ and $\boldsymbol{\Upsilon}$, respectively. More precisely, $\frac{\sigma_{3}^{2}}{\sigma_{i}^{2}} \mathbf{H}_{i 3}^{\dagger} \mathbf{H}_{i 3}=\mathbf{V}_{\bar{i}} \operatorname{diag}\left(\mathbf{h}_{\bar{i}}\right) \mathbf{V}_{\bar{i}}^{\dagger}$ and $\dot{\boldsymbol{\Upsilon}}_{i}=\mathbf{U}_{i} \operatorname{diag}\left(\boldsymbol{v}_{i}\right) \mathbf{U}_{i}^{\dagger}, i=$ 1,2 , where $\mathbf{h}_{i}$ and $\boldsymbol{v}_{i}$ are vectors containing the eigenvalues of $\frac{\sigma_{3}^{2}}{\sigma_{i}^{2}} \mathbf{H}_{i 3}^{\dagger} \mathbf{H}_{i 3}$ and $\dot{\Upsilon}_{i}$, correspondingly, sorted in descending order. In addition, we define $\mathbf{h}=\left[\mathbf{h}_{1} \mathbf{h}_{2}\right]=\left[h_{1}, h_{2}, \ldots h_{2 n_{3}}\right]$ and $\boldsymbol{v}=\left[\boldsymbol{v}_{1} \boldsymbol{v}_{2}\right]=\left[v_{1}, v_{2}, \ldots v_{2 n_{3}}\right]$. Meanwhile, $\widehat{\mathbf{G}}$ is a diagonal matrix containing the eigenvalues of $\overline{\mathbf{G}}^{\dagger} \overline{\mathbf{G}}$ sorted in descending order, such that $\widehat{\mathbf{G}}=\operatorname{diag}(\mathrm{g})$, with $\mathbf{g}=$

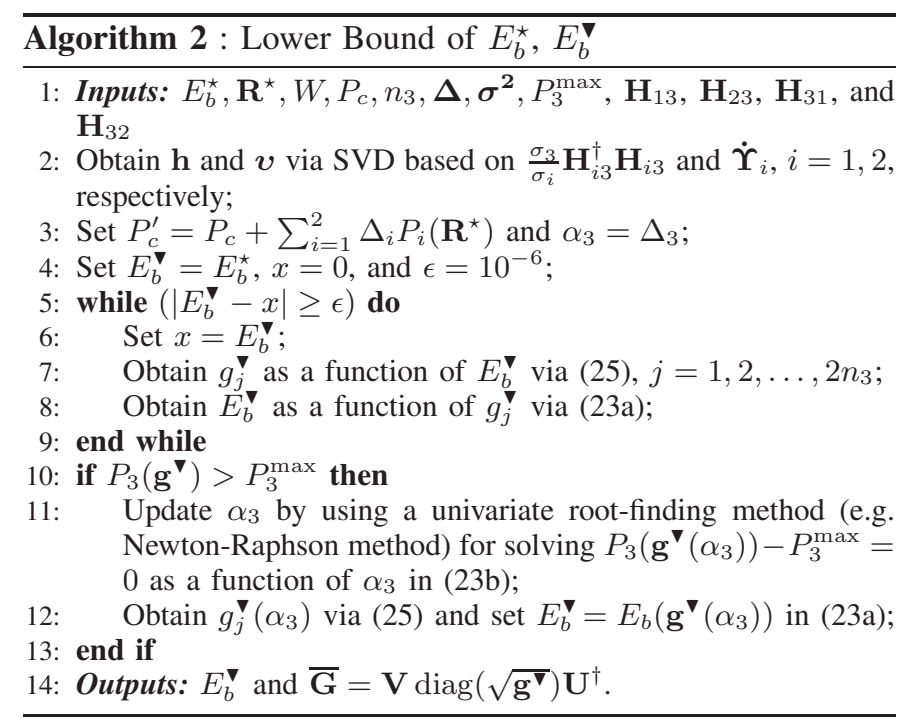

$\left[g_{1}, g_{2}, \ldots g_{2 n_{3}}\right]$. By inserting (22) into (19a), (19b) and (20), the relaxed form of (19) simplifies as

$$
\begin{aligned}
& \min _{\mathbf{g}} E_{b}(\mathbf{g})=\frac{P_{c}^{\prime}+\Delta_{3} P_{3}(\mathbf{g})}{R_{\Sigma}(\mathbf{g})} \\
& \text { s.t. } P_{3}(\mathbf{g})=\sigma_{3}^{2} \sum_{j=1}^{2 n_{3}} g_{j}\left(1 / 2+v_{j}\right) \leq P_{3}^{\max },
\end{aligned}
$$

with

$$
R_{\Sigma}(\mathbf{g})=W \sum_{j=1}^{2 n_{3}} \log _{2}\left(1+\frac{g_{j} h_{j} v_{j}}{1+g_{j} h_{j}}\right) .
$$

Accordingly, the elements of the optimal argument of (23), i.e. $\mathbf{g}^{\mathbf{\nabla}}=\left[g_{1}^{\mathbf{\nabla}}, g_{2}^{\mathbf{\nabla}}, \ldots, g_{2 n_{3}}^{\mathbf{\nabla}}\right]$ can be obtained in closed-form as

$$
g_{j}^{\boldsymbol{\nabla}}=\frac{1}{2 h_{j}\left(1+v_{j}\right)}\left[\sqrt{v_{j}^{2}+\frac{8 W E_{b}^{\mathbf{\nabla}} v_{j} h_{j}\left(1+v_{j}\right)}{\ln (2) \alpha_{3} \sigma_{3}^{2}\left(1+2 v_{j}\right)}}-v_{j}-2\right],
$$

$j=1,2, \ldots, 2 n_{3}$, where $E_{b}^{\mathbf{v}}=E_{b}\left(\mathbf{g}^{\mathbf{\nabla}}\right)$ is the optimal solution of (23), $\alpha_{3}=\delta_{3}^{\mathbf{V}} \Delta_{3}+\left(1-\delta_{3}^{\mathbf{v}}\right) \mu_{3}^{\mathbf{v}} \geq 0$, with $\mu_{3}^{\mathbf{\nabla}} \geq 0$ being a Lagrangian multiplier and $\delta_{3}^{\mathbf{v}}=1$ if $P_{3}\left(\mathbf{g}^{\boldsymbol{\nabla}}\right) \leq P_{3}^{\max }$ or else $\delta_{3}^{\mathbf{V}}=0$. See proof in Section D of the Appendix.

Similar to finding $\mathbf{R}^{\star}$ in Section III-B, we first need to find $\mathrm{g}^{\mathbf{v}}$ in the unconstrained case before refining the values of its elements if $P_{3}\left(\mathbf{g}^{\boldsymbol{\nabla}}\right)>P_{3}^{\max }$, as it is detailed in Algorithm 2. In the unconstrained case, solving (23) is equivalent to solving a single equation, i.e. $E_{b}^{\mathbf{V}}=E_{b}\left(\mathbf{g}=\mathbf{g}^{\mathbf{v}}\right)$, with one unknown, i.e. $E_{b}^{\mathbf{v}}$; this can be done in a low-complexity manner by using a classic univariate root-finding algorithm, as it is shown in Algorithm 2. Whereas, in the case that $P_{3}\left(g^{\mathbf{\nabla}}\right)>P_{3}^{\max }$, solving (23) is still about solving a single equation, i.e. $P_{3}\left(\mathbf{g}^{\mathbf{\nabla}}\left(\mu_{3}^{\mathbf{\nabla}}\right)\right)=P_{3}^{\max }$, with one unknown, i.e. $\mu_{3}^{\mathbf{\nabla}}$. This is similar to a water-filling problem that can be solved in a lowcomplexity manner by using a simple root-finding algorithm (e.g. Newton-Raphson method [35]). Note that Algorithm 2 follows the same design principle as Algorithm 1, but in a simpler form (without requiring recursive functions), given that (19) or (23) has only one power constraint instead of three in (11). 


\section{B. Upper bound to the optimal solution of (17)}

An upper bound of $E_{b}^{\star}$ can simply be obtained by using classic convex optimization methods [34], e.g. gradient/projected gradient search, log-barrier, or Newton method, to solve (17). Indeed, if (17) was at least pseudo-convex, then applying such a method for solving (17) would return $E_{b}^{\star}$. Given that (17) is not pseudo-convex, solving (17) by using classic convex optimization tools will generally return a sub-optimal solution $E_{b}^{\mathbf{\Delta}}=E_{b}\left(\mathbf{G}^{\mathbf{\Lambda}}\right)$, such that $E_{b}^{\star} \leq E_{b}^{\mathbf{\Delta}}$.

\section{Solving (17) and finding $\mathbf{G}^{\star}$}

In order to find the optimal solution of (17), we leverage on $E_{b}^{\mathbf{V}}$ and $E_{b}^{\mathbf{\Delta}}$ (discussed in Sections IV-A and IV-B, respectively) as well as the following proposition

Proposition 6: Let $E_{b}^{\mathbf{V}}+\varepsilon^{\star}=E_{b}^{\star}, \varepsilon^{\star} \geq 0$, such that

$$
\left\{\begin{array}{l}
E_{b}^{\mathbf{\nabla}}+\varepsilon<E_{b}^{\star}, \quad \text { if } \varepsilon \in\left[0, \varepsilon^{\star}\right) ; \\
E_{b}^{\boldsymbol{\nabla}}+\varepsilon \geq E_{b}^{\star}, \quad \text { if } \varepsilon \in\left[\varepsilon^{\star}, E_{b}^{\boldsymbol{\Delta}}-E_{b}^{\mathbf{\nabla}}\right] .
\end{array}\right.
$$

Given that $E_{b}(\mathbf{G})=P_{\Sigma}(\mathbf{G}) / R_{\Sigma}(\mathbf{G})$, it then exists matrices $\mathbf{G} \in \mathcal{D}_{E_{b}}\left(\mathcal{D}_{E_{b}}\right.$ being the domain of $\left.E_{b}\right)$ that verifies

$$
f(\mathbf{G})=P_{\Sigma}(\mathbf{G})-\left(E_{b}^{\mathbf{V}}+\varepsilon\right) R_{\Sigma}(\mathbf{G})=0,
$$

if and only if $\varepsilon \geq \varepsilon^{\star}$.

Proof: Since $E_{b}(\mathbf{G})=P_{\Sigma}(\mathbf{G}) / R_{\Sigma}(\mathbf{G})$, then $P_{\Sigma}(\mathbf{G})-$ $E_{b}(\mathbf{G}) R_{\Sigma}(\mathbf{G})=0$. Given that $E_{b}^{\star}=E_{b}\left(\mathbf{G}^{\star}\right)$ is the global minimum of $E_{b}$, i.e. the lowest possible value of the codomain of $E_{b}, E_{b}^{\mathbf{V}}+\varepsilon$ does not belong to the codomain of $E_{b}$ when $E_{b}^{\mathbf{V}}+\varepsilon<E_{b}^{\star}$, such that (26) has no solution in $\mathcal{D}_{E_{b}}$. Indeed if $E_{b}^{\mathbf{V}}+\varepsilon<E_{b}^{\star}$, then $P_{\Sigma}(\mathbf{G}) / R_{\Sigma}(\mathbf{G})>E_{b}^{\mathbf{V}}+\varepsilon \Leftrightarrow P_{\Sigma}(\mathbf{G})-$ $\left(E_{b}^{\mathbf{\nabla}}+\varepsilon\right) R_{\Sigma}(\mathbf{G})>0, \forall \mathbf{G} \in \mathcal{D}_{E_{b}}$.

Consequently, according to Proposition 5 , if $\varepsilon$ in (26) were to increase in an infinitesimal manner from 0 to $E_{b}^{\mathbf{\Delta}}-E_{b}^{\mathbf{V}}$ then the first value of $\varepsilon$ for which it would exist a matrix $\mathbf{G} \in$ $\mathcal{D}_{E_{b}}$ that verifies (26) would be $\varepsilon^{\star}$ and its associated matrix would be $\mathbf{G}^{\star}$. In the case that $P_{3}\left(\mathbf{G}^{\star}\right) \leq P_{3}^{\max }$ (unconstrained optimization), then $\varepsilon^{\star}$ and $\mathbf{G}^{\star}$ would also verify

$$
\nabla_{\mathbf{G}} P_{\Sigma}\left(\mathbf{G}^{\star}\right)-\left(E_{b}^{\mathbf{\nabla}}+\varepsilon^{\star}\right) \nabla_{\mathbf{G}} R_{\Sigma}\left(\mathbf{G}^{\star}\right)=\mathbf{0},
$$

where $\nabla_{\mathbf{G}} R_{\Sigma}(\mathbf{G})=\frac{2 W}{\ln (2)} \sum_{i=1}^{2} \mathbf{H}_{\bar{i} 3}^{\dagger}\left[u_{i}\left(\mathbf{G}\left(\mathbf{I}_{n_{3}}+\dot{\boldsymbol{\Upsilon}}_{i}\right)^{1 / 2}\right) \mathbf{H}_{\bar{i} 3}\right.$ $\left.\times \mathbf{G}\left(\mathbf{I}_{n_{3}}+\dot{\boldsymbol{\Upsilon}}_{i}\right)-u_{i}(\mathbf{G}) \mathbf{H}_{\bar{i} 3} \mathbf{G}\right]$ and $\nabla_{\mathbf{G}} P_{\Sigma}(\mathbf{G})=\nabla_{\mathbf{G}} P_{3}(\mathbf{G})$ $=2 \Delta_{3} \mathbf{G}\left(\mathbf{I}_{n_{3}}+\sum_{i=1}^{2} \dot{\boldsymbol{\Upsilon}}_{i}\right)$, based on (17a) and matrix calculus [36]. In addition, $u_{i}(\mathbf{X})=\left(\mathbf{I}_{n_{\bar{i}}}+\frac{\sigma_{3}^{2}}{\sigma_{\bar{i}}^{2}} \mathbf{H}_{\bar{i} 3} \mathbf{X} \mathbf{X}^{\dagger} \mathbf{H}_{\bar{i} 3}^{\dagger}\right)^{-1}$. See the proof for (27) in Section $\mathrm{E}$ of the Appendix. Accordingly, a simple method for finding $\mathbf{G}^{\star}$ would be to increase $\varepsilon$ from 0 to $E_{b}^{\boldsymbol{\Delta}}-E_{b}^{\mathbf{V}}$ (in very small steps) in (26) and use a generic non-linear equation solver (e.g. "fsolve" in Matlab) to solve (26), until the left-hand side of (26) is equal to (or very close to) zero. In order to reduce the number of $\varepsilon$ values that are evaluated in (26), a bisection search method (with 0 and $E_{b}^{\boldsymbol{\Delta}}-E_{b}^{\boldsymbol{V}}$ as initial endpoint values) could be used instead of increasing $\varepsilon$ in regular steps, as it is detailed in see Algorithm 3. Note that the computational complexity of the bisection method and of any non-linear equation solver is inherent to its initial search values. By using fairly tight bounds of $E_{b}^{\star}$ as initial values for the bisection search and $\mathbf{G}^{\boldsymbol{\Delta}}$

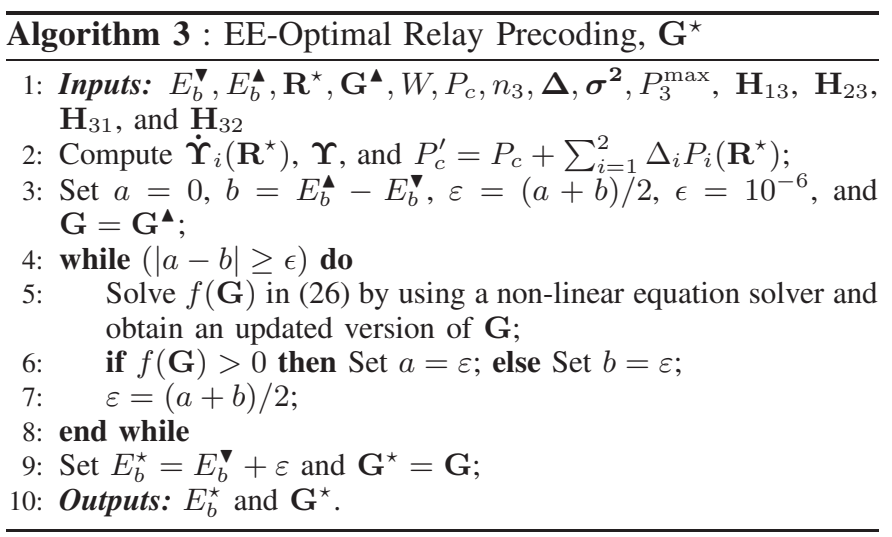

as the initial value for solving (26), it ensures a good balance between computational complexity and convergence speed. Indeed, since the main source of complexity in Algorithm 3 (i.e. especially for large value of $n_{3}$ ) is to find a solution to (26), it is important to reduce the number of iterations of the bisection method by choosing suitable initial values.

\section{Joint Source and Relay Precoding Optimization}

\section{A. Alternating Optimization Procedure}

Similar to the works in [9], [16], [19], [24], the optimization problem in (10) can be solved by using an alternating optimization procedure based on $\mathbf{R}^{\star}$ and $\mathbf{G}^{\star}$, as follows:

1) Set $\mathbf{G}^{\star^{(0)}}=\mathbf{G}^{(0)}$, where $\mathbf{G}^{(0)}$ is an initialization matrix. Note that as in [9], we consider $N$ different randomly selected initialization matrices.

2) Set $\mathbf{R}^{\star^{(0)}}=\mathbf{I}_{n_{0}}$ and obtain $E_{b}^{\star}$ by inserting both $\mathbf{R}^{\star}$ as well as $\mathbf{G}^{\star}$ in (9).

3) At the $k$-th iteration, $\mathbf{G}^{\star^{(k)}}$ and $E_{b}^{\star}$ are used in Algorithm 1 to update the value of $E_{b}^{\star}$ and return $\mathbf{R}^{\star^{(k+1)}}$.

4) Next, $\mathbf{R}^{\star^{(k+1)}}$ and $E_{b}^{\star}$ are used in Algorithm 3 to update the value of $E_{b}^{\star}$ and return $\mathbf{G}^{\star^{(k+1)}}$.

5) Steps 3) and 4) are repeated iteratively until convergence, i.e. until the values of $E_{b}^{\star}$ at the end of the $k$-th and $k+1$-th iterations are the same.

6) Steps 1) to 5) are repeated $N$ times for each randomly selected initialization matrix $\mathbf{G}^{(0)}$; the outcome of this procedure is the lowest $E_{b}^{\star}$ (out of the $N$ final $E_{b}^{\star}$ values) and its corresponding $\mathbf{R}^{\star}$ and $\mathbf{G}^{\star}$ matrices.

\section{B. Convergence Discussion and Results}

The monotonic convergence of the above alternating procedure for solving (10) can be proved by following the same line of reasoning as in [24]. On the one hand, since $\mathbf{R}^{\star}$ is the optimal argument of (10) when $\mathbf{G}$ is fixed, it implies that

$$
E_{b}\left(\mathbf{R}^{\star^{(k+1)}}, \mathbf{G}^{\star^{(k)}}\right) \leq E_{b}\left(\mathbf{R}^{\star^{(k)}}, \mathbf{G}^{\star^{(k)}}\right) .
$$

On the other hand, since $\mathbf{G}^{\star}$ is the optimal argument of (10) when $\mathbf{R}$ is fixed, it implies that

$$
E_{b}\left(\mathbf{R}^{\star^{(k+1)}}, \mathbf{G}^{\star^{(k+1)}}\right) \leq E_{b}\left(\mathbf{R}^{\star^{(k+1)}}, \mathbf{G}^{\star^{(k)}}\right) .
$$

By combining (28) and (29), it then implies that

$$
E_{b}\left(\mathbf{R}^{\star^{(k+1)}}, \mathbf{G}^{\star^{(k+1)}}\right) \leq E_{b}\left(\mathbf{R}^{\star^{(k)}}, \mathbf{G}^{\star^{(k)}}\right) .
$$


TABLE I

COEFFICIENT OF VARIATION, $c_{v}$ (IN \%), OF THE FINAL VALUE OF $E_{b}^{\star}$ FOR $N=100$ DIFFERENT RANDOM INITIALIZATION MATRICES

\begin{tabular}{|c|c|c|c|c|c|c|c|c|}
\hline & \multicolumn{7}{|c|}{$\sigma_{3}^{2}(\mathrm{dBW})$} \\
\hline & & -30 & -20 & -10 & 0 & 10 & 20 & 30 \\
\hline \multirow{4}{*}{$\begin{array}{l}\sigma_{1}^{2}, \sigma_{2}^{2} \\
(\mathrm{dBW})\end{array}$} & -30 & $0.018 \%$ & $0.025 \%$ & $0.032 \%$ & $0.043 \%$ & $0.11 \%$ & $0.18 \%$ & $0.21 \%$ \\
\hline & -10 & $0.021 \%$ & $0.022 \%$ & $0.027 \%$ & $0.052 \%$ & $0.16 \%$ & $0.31 \%$ & $0.54 \%$ \\
\hline & 10 & $0.017 \%$ & $0.021 \%$ & $0.031 \%$ & $0.047 \%$ & $0.12 \%$ & $0.25 \%$ & $0.43 \%$ \\
\hline & 30 & $0.015 \%$ & $0.020 \%$ & $0.025 \%$ & $0.038 \%$ & $0.09 \%$ & $0.27 \%$ & $0.48 \%$ \\
\hline
\end{tabular}

In other words, equation (30) indicates that the conditional updating of $\mathbf{R}^{\star}$ (for a fixed $\mathbf{G}^{\star}$ ) and $\mathbf{G}^{\star}$ (for a fixed $\mathbf{R}^{\star}$ ) at each iteration either decreases or maintains the value of $E_{b}^{\star}=E_{b}\left(\mathbf{R}^{\star}, \mathbf{G}^{\star}\right) ; E_{b}^{\star}$ being the best local minimum of $E_{b}$, for a given $\mathbf{R}^{\star}$ or $\mathbf{G}^{\star}$, at each iteration. However, it is important to note, as for instance in [9], [19], that $E_{b}^{\star}$ is not necessarily always the global minimum of (10) in the general case since $E_{b}(\mathbf{R}, \mathbf{G})$ in (10) is not necessarily jointly pseudoconvex/convex in both $\mathbf{R}$ and $\mathbf{G}$. Nevertheless, in some special cases, $E_{b}^{\star}$ is guaranteed to be the global minimum of (10). For instance, if both $\sigma_{1}^{2} \ll \sigma_{3}^{2}$ and $\sigma_{2}^{2} \ll \sigma_{3}^{2}$, then the rate equation in (3) becomes independent of $\mathbf{G}$ and, in turn, $\mathbf{R}$ and $\mathbf{G}$ can be decorrelated. According to [9] (for the one way scenario), this is a sufficient condition for (10) to converge to a global optimum. Meanwhile, if $\sigma_{1}^{2} \gg \sigma_{3}^{2}$ or $\sigma_{2}^{2} \gg \sigma_{3}^{2}$, then the rate equation in (3) becomes independent of $\mathbf{R}_{1}$ or $\mathbf{R}_{2}$ and, in turn, (10) becomes a one-way joint optimization problem instead of a two-way problem. A problem that has been optimally solved in [10].

In order to better understand the convergence behavior of the alternating optimization procedure, we have reported in Table I the coefficient of variation, $c_{v}$, of the final $E_{b}^{\star}$ value (i.e. the value of $E_{b}^{\star}$ at the end of step 5) of this procedure) for $N=100$ different random initialization matrices $\mathbf{G}^{(0)}$ and various $\sigma_{i}^{2}$ values, $i=1,2,3$. The coefficient of variation is a standardized tool for measuring the dispersion of random variable distributions that is defined as a ratio (in \%) between the standard deviation and the mean of random variables; a very low $c_{v}$ value indicates a high similarity between random variables. Here, we report the ratio between the standard deviation and the mean of the $N=100$ final values of $E_{b}^{\star}$, averaged over 1000 runs for each $\sigma_{i}^{2}$ setting. The results show that the values of $c_{v}$ are quite low $(<0.1 \%)$ for many settings. In turn, it indicates that the final values of $E_{b}^{\star}$ are likely to be similar regardless of the initialization matrix $\mathbf{G}^{(0)}$ for those settings, such that our algorithm is likely to converge towards the global minimum of (10). In order to complement these results, we plot in Fig. 2 a snapshot of the convergence behavior of the alternating procedure as a function of the number of iterations for $N=10$ in two different settings; a very low and a low $c_{v}$ setting (based on Table I), i.e. $\sigma_{1}^{2}=\sigma_{2}^{2}=\sigma_{3}^{2}=-30 \mathrm{dBW}$ and $\sigma_{1}^{2}=\sigma_{2}^{2}=\sigma_{3}^{2}=30 \mathrm{dBW}$, respectively. Each line on the plot shows the variation of $E_{b}^{\star}$ (i.e. the value of $E_{b}^{\star}$ at the end of step 4) of the alternating procedure after each iteration) for a given initialisation matrix $\mathbf{G}^{(0)}$. Firstly, these results confirm the monotonic convergence

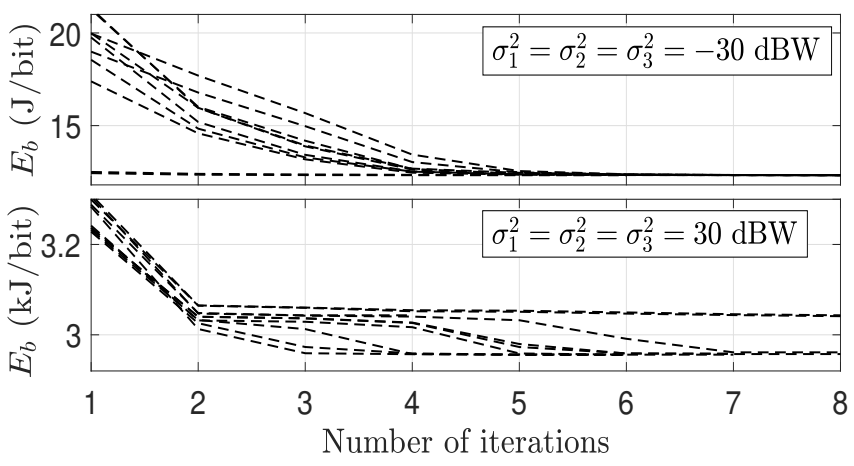

Fig. 2. Convergence behavior of our alternating optimization procedure as a function of the number of iterations for $N=10$ and two different settings.

of the alternating procedure that has been proved in (30). It also confirms that the final value of $E_{b}^{\star}$ (after several iterations) is hardly affected by $\mathbf{G}^{(0)}$ when $c_{v}$ is very low. Whereas in the case of $\sigma_{1}^{2}=\sigma_{2}^{2}=\sigma_{3}^{2}=30 \mathrm{dBW}$, different initialization matrices can result in different outcomes, i.e. two different final values of $E_{b}^{\star}$ in the lower part of Fig. 2. Hence, as in [9], the use of multiple initialization matrices increase the likelihood of reaching the global minimum of (10).

\section{NUMERICAL RESULTS AND DisCUSSIONS}

Our simulation results are obtained based on the following assumptions:

- We consider five different node, antenna and power settings that are detailed in Table II. Settings $\mathbf{A}$ and $\mathbf{B}$ are unconstrained settings (i.e. which is equivalent to the case of $\left.P_{j}^{\max } \rightarrow \infty, j=1,2,3\right)$, while Settings $\mathbf{C}, \mathbf{D}$ and $\mathbf{E}$ rely on realistic power constraint values at each node.

- We use the power parameter values of Table I of [20] for setting the values of $\Delta_{\text {. }}, P^{\mathrm{CipA}}$, and $P^{\mathrm{Ci}}$ in Section II-B. Moreover, note that $P^{\max }$ per antenna is around 20 $\mathrm{W}$ for a typical macro BS [27], and between 1 to $5 \mathrm{~W}$ for a typical urban/rural relay [28]. Furthermore, we set $\varsigma=1 / 2$ in $P_{c}$ in (6).

- We consider a single-tap i.i.d MIMO Rayleigh fading channel between each node, assume $N=10$ in step 1) of Section V-A), and set $W=1$ without loss of generality.

In order to demonstrate the benefit of our energy efficient precoding scheme for the two-way MIMO-AF relay scenario, we benchmark it against the most relevant existing one-way and two-way MIMO-AF schemes, e.g. [9], [10], [16], [19]. We also evaluate the relative tightness of the lower and upper bounds of the relay optimization process (introduced in Section IV-A and IV-B) with the optimal process of Section IV-C. In the following, for ease of introduction, we rely on the subsequent notation to refer to our scheme and the relevant schemes it is benchmarked against:

- $E_{b}^{\star}=E_{b}\left(\mathbf{R}^{\star}, \mathbf{G}^{\star}\right)$ is the outcome of the joint source and relay optimization procedure of Section V-A, based on our EE-optimal source and relay precoding matrices.

- $E_{b}^{\mathbf{V}}$ is the outcome of the optimization procedure of Section V-A, but where Algorithm 3 is replaced with our lower bound relay optimization method of Algorithm 2. 
TABLE II

SIMULATION SETTINGS (TYPE OF NODES, NUMBER OF ANTENNAS AND POWER PARAMETER VALUES)

\begin{tabular}{|c||c|c|c|c|c|c|c|c|c|c|c|c|c|c|c|c|c|}
\hline Settings & $\begin{array}{c}\text { Node } \\
1\end{array}$ & $\begin{array}{c}\text { Node } \\
2\end{array}$ & $n_{1}$ & $n_{2}$ & $n_{3}$ & $\Delta_{1}$ & $\Delta_{2}$ & $\Delta_{3}$ & $\begin{array}{c}P_{1}^{\mathrm{Cip}} \\
(\mathrm{W})\end{array}$ & $\begin{array}{c}P_{2}^{\mathrm{Cip}} \\
(\mathrm{W})\end{array}$ & $\begin{array}{c}P_{3}^{\mathrm{Cip}} \\
(\mathrm{W})\end{array}$ & $\begin{array}{c}P_{1}^{\mathrm{Ci}} \\
(\mathrm{W})\end{array}$ & $\begin{array}{c}P_{2}^{\mathrm{Ci}} \\
(\mathrm{W})\end{array}$ & $\begin{array}{c}P_{3}^{\mathrm{Ci}} \\
(\mathrm{W})\end{array}$ & $\begin{array}{c}P_{1}^{\max } \\
(\mathrm{W})\end{array}$ & $\begin{array}{c}P_{2}^{\max } \\
(\mathrm{W})\end{array}$ & $\begin{array}{c}P_{3}^{\max } \\
(\mathrm{W})\end{array}$ \\
\hline $\mathbf{A}$ & BS & UE & 4 & 4 & 4 & 4.7 & 2 & 6.3 & 100 & 0.03 & 4.0 & 180 & 0.07 & 4.9 & $\infty$ & $\infty$ & $\infty$ \\
\hline B & UE & UE & 4 & 4 & 4 & 2 & 2 & 6.3 & 0.03 & 0.03 & 4.0 & 0.07 & 0.07 & 4.9 & $\infty$ & $\infty$ & $\infty$ \\
\hline C & BS & UE & 4 & 4 & 4 & 4.7 & 2 & 6.3 & 100 & 0.03 & 4.0 & 180 & 0.07 & 4.9 & 80 & 0.8 & 10 \\
\hline D & UE & UE & 4 & 4 & 4 & 2 & 2 & 6.3 & 0.03 & 0.03 & 4.0 & 0.07 & 0.07 & 4.9 & 0.8 & 0.8 & 10 \\
\hline E & BS & UE & 5 & 3 & 2 & 4.7 & 2 & 6.3 & 100 & 0.03 & 4.0 & 180 & 0.07 & 4.9 & 100 & 0.6 & 5 \\
\hline
\end{tabular}

- $E_{b}^{\mathbf{\Delta}}$ is the outcome of the optimization procedure of Section V-A, but where Algorithm 3 is replaced by a generic gradient/projected gradient search algorithm.

- $E_{b}^{\mathrm{TWB}}$ is the outcome of the EE-based source and relay beamforming method proposed in [19] for the two-way MIMO-AF relay channel scenario, where TWB stands for two-way beamforming.

- $\max$ SE is the outcome of the SE-based source and relay precoding method proposed in [16] for the two-way MIMO-AF relay channel scenario.

- $E_{b}^{\text {AOW }}$ is the aggregated outcome of the EE-optimal source and relay precoding method proposed in [9], [10] for the one-way MIMO-AF relay channel scenario, where AOW stands for aggregated one-way. In other words, we take into account both Node $1 \rightarrow \mathrm{RN} \rightarrow$ Node 2 and Node $2 \rightarrow \mathrm{RN} \rightarrow$ Node 1 transmissions, each of them having two transmission phases, and we assume that they happen in parallel over two different frequency bands, i.e. by assuming frequency division duplexing, to ensure a fair comparison (over the same number of transmission phases) with the two-way MIMO-AF schemes.

Note that $P_{c}$, which accounts for all the fixed circuit consumed powers in the system, is different for the one-way or two-way relay channel. Indeed, $P_{c}$ is defined as in (6) for the two-way scenario, whereas, according to [31], $P_{c}=n_{i} P_{i}^{\mathrm{CipA}}+P_{i}^{\mathrm{Ci}}+$ $\varsigma\left(n_{\bar{i}} P_{\bar{i}}^{\mathrm{CipA}}+P_{\bar{i}}^{\mathrm{Ci}}\right)+n_{1} P_{1}^{\mathrm{SlpA}}+n_{2} P_{2}^{\mathrm{SlpA}}+(1+\varsigma)\left(n_{3} P_{3}^{\mathrm{CipA}}+P_{3}^{\mathrm{Ci}}\right)$ in the one-way scenario, where $P_{i}^{\mathrm{SlpA}}, i=1,2$ is the power consumed when Node 1 or 2 is in sleep mode. This needs to be taken into account when comparing one-way with two-way schemes to ensure a fair comparison.

Figure 3 compares the sum-rate and EE performances of our scheme against those of the SE-based two-way precoding scheme of [16] (max SE) and EE-based two-way beamforming scheme of [19] ( $E_{b}^{\mathrm{TWB}}$ ) in Settings $\mathbf{C}$ and $\mathbf{D}$ for $\sigma_{1}^{2}=\sigma_{2}^{2}=$ $\sigma_{3}^{2}=\sigma^{2}$. Note that the unit for sum-rate is bit/2/s, since we consider the number of transmitted bits over two transmission phases. The results first show that max SE exhibits the best sum-rate performance. Whereas our scheme can provide more than twice the rate of $E_{b}^{\mathrm{TWB}}$. In terms of energy consumption, the results confirm that our scheme is the best; it can reduce $E_{b}$ by up to $38 \%$ and $50 \%$ in comparison with max SE and $E_{b}^{\mathrm{TWB}}$, respectively, in good channel conditions (e.g. when $\sigma^{2}=-20$ $\mathrm{dB})$, but at the expense of a sum-rate reduction of up to $20 \%$ when compared to max SE. Given that max SE is meant by design to be SE-optimal and our scheme is EE-optimal,

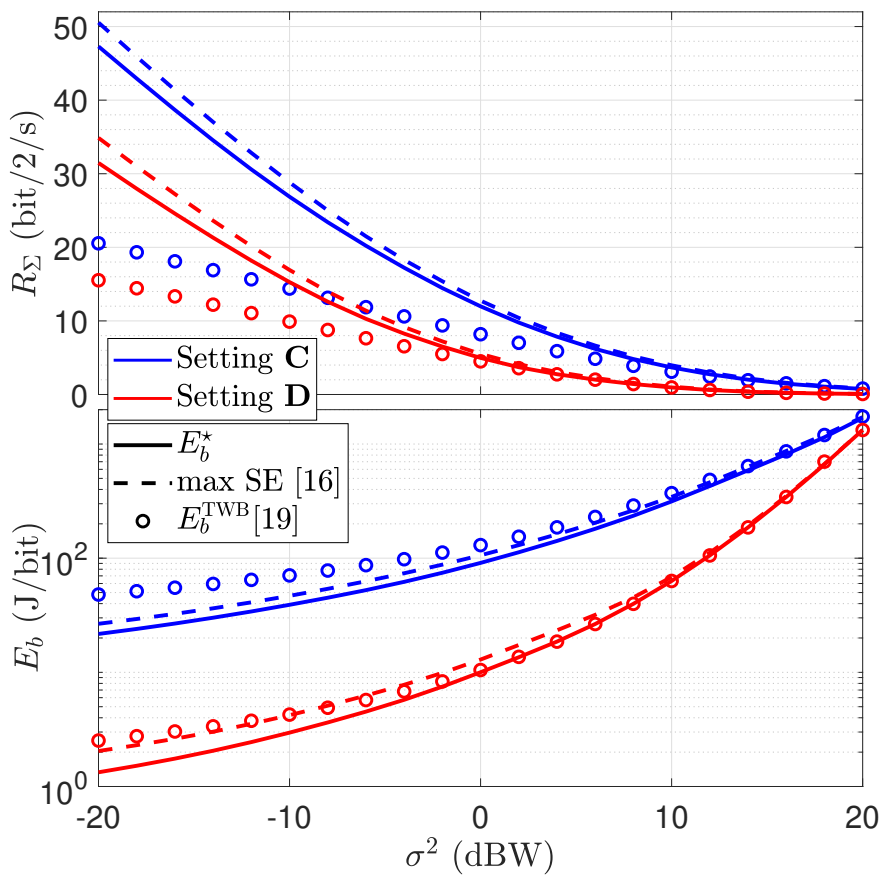

Fig. 3. Sum-rate and EE performances of our scheme, against two other relevant existing two-way MIMO-AF precoding schemes, as a function of $\sigma^{2}$ in $\mathrm{dBW}$.

it is expected that max SE would perform better than our scheme in terms of sum-rate, and vice-versa in terms of energy consumption. However, it is interesting to see that our scheme outperforms $E_{b}^{\mathrm{TWB}}$ (EE-based scheme of [19]), both in terms of energy and rate. Contrary to $E_{b}^{\mathrm{TWB}}$, our scheme can take full advantage of having $n_{i}$ transmit antennas at Nodes $i, i=1,2$; it can simultaneously transmit up to $n_{i}$ streams of information instead of 1 (the best channel eigenmode out of $n_{i}$ ) in $E_{b}^{\mathrm{TWB}}$. Moreover, our relay precoding matrix is EE-optimal, while the one used in $E_{b}^{\mathrm{TWB}}$ is only sub-optimal (i.e. it is based on an approximation; see [19] for more details). Finally, it can also be remarked that the performances of the three schemes converge at high $\sigma^{2}$, such that the type of precoding design becomes less of an issue in poor channel conditions.

Figure 4 compares the transmit power (in both transmission phases), sum-rate and EE performances of our scheme against those of the EE-based two-way beamforming scheme of [19] $\left(E_{b}^{\mathrm{TWB}}\right)$ and the EE-based one-way precoding scheme of [9], [10] $\left(E_{b}^{\mathrm{AOW}}\right)$ in Settings $\mathbf{A}$ and $\mathbf{C}$ for $\sigma_{1}^{2}=\sigma_{2}^{2}=\sigma_{3}^{2}=\sigma^{2}$. Firstly, it can be noted, as explained in Section III-B, that all 


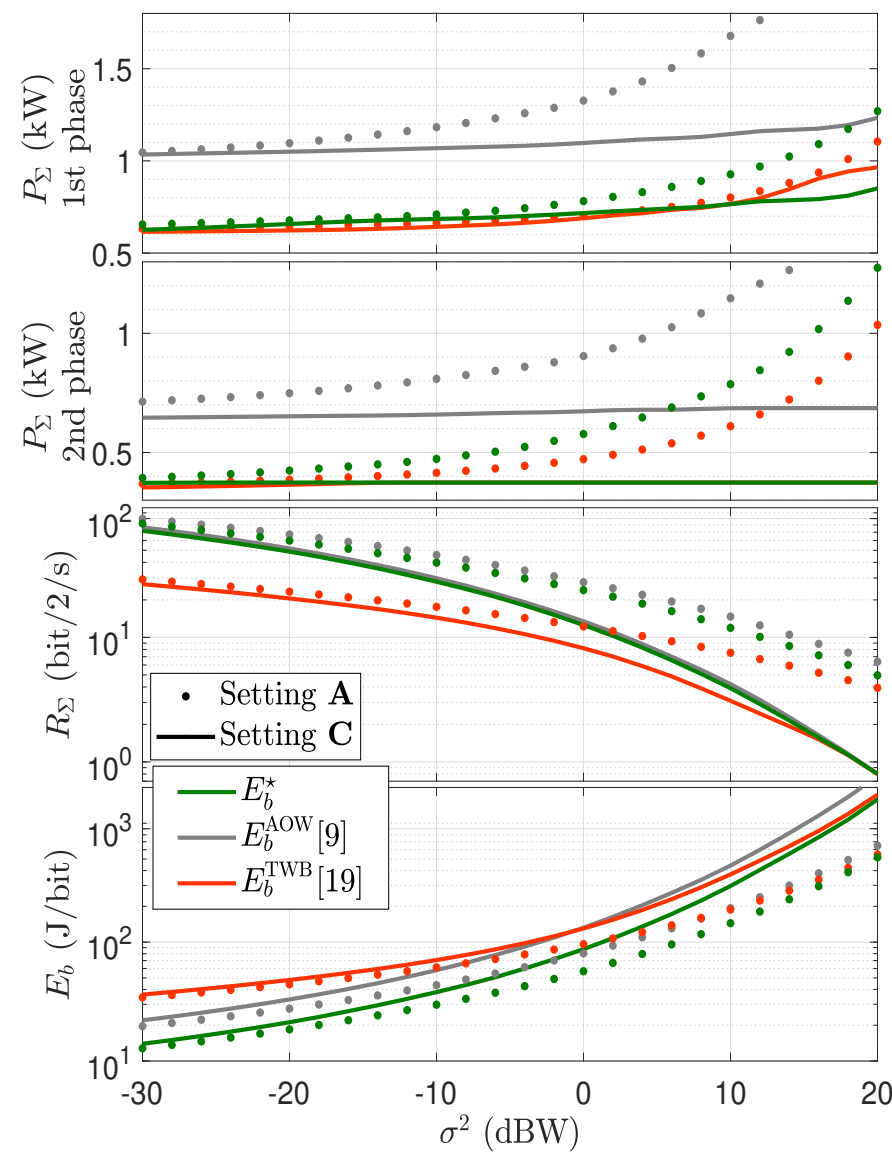

Fig. 4. Transmit power, sum-rate, and EE performances of our scheme against other relevant existing EE-based MIMO AF schemes, as a function of $\sigma^{2}$ in dBW.

the schemes perform better in terms of $E_{b}$ in the unconstrained rather than in the power constrained scenario. Secondly, the results clearly indicate that our scheme outperforms $E_{b}^{\mathrm{AOW}}$ and $E_{b}^{\mathrm{TWB}}$ by up to $35 \%$ and $60 \%$, respectively, in terms of $E_{b}$ for both the unconstrained and power constrained scenarios. Given that $E_{b}$ is a ratio between power and rate, insights on our scheme significant $E_{b}$ gains over $E_{b}^{\mathrm{AOW}}$ and $E_{b}^{\mathrm{TWB}}$ can be drawn by analyzing the rate and power performances of all the schemes. We already know from Fig. 3 that the rate of $E_{b}^{\mathrm{TWB}}$ is significantly lower than the rate of our scheme. Here, we can also remark that it consumes more or less the same power as our scheme in both transmission phases (in the power constrained case). Hence, the key contributing factor behind the large $E_{b}$ gain of our scheme over $E_{b}^{\mathrm{TWB}}$ is its better rate performance. Whereas, as far as $E_{b}^{\mathrm{AOW}}$ is concerned, its rate performance is slightly better than that of our scheme (bearing in mind that it uses twice the bandwidth as our scheme), but its power consumption is significantly worse in both phases (our scheme consumes roughly between $30-40 \%$ less power than $E_{b}^{\mathrm{AOW}}$ ). Indeed, in one-way transmission, Nodes 1 and 2 operate in sleep mode during some of the transmission phases (see [10] for more details); this increases the power consumption and in turn $E_{b}$, when compared to a two-way transmission. Meanwhile, jointly optimizing the two transmission ways is more efficient than optimizing each way separately. These two factors explain the large $E_{b}$ gain of our

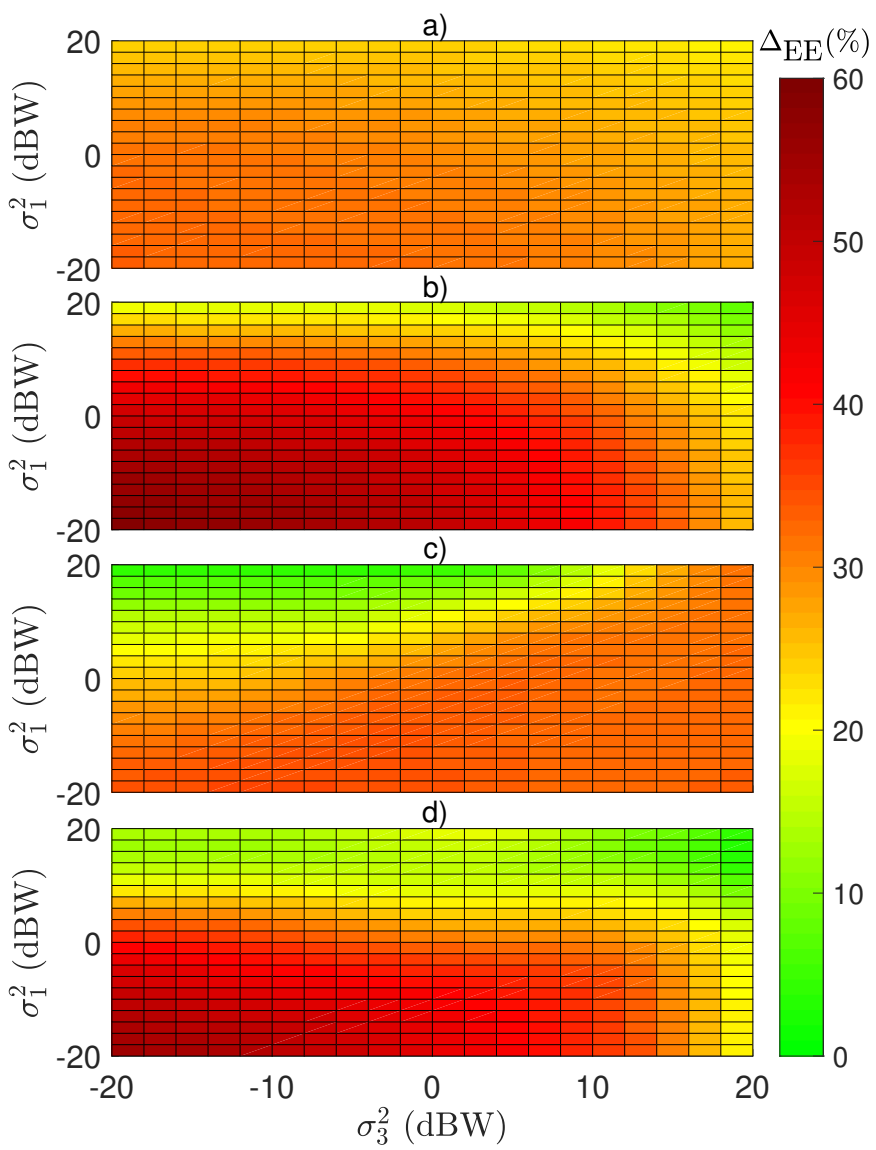

Fig. 5. EE gain comparison of our scheme against a) $E_{b}^{\mathrm{AOW}}$ in Setting $\mathbf{A}$, b) $E_{b}^{\mathrm{TWB}}$ in Setting $\mathbf{A}$, c) $E_{b}^{\mathrm{AOW}}$ in Setting $\mathbf{C}$, and d) $E_{b}^{\mathrm{TW}}$ in Setting $\mathbf{C}$, as a function of $\sigma_{1}^{2}$ and $\sigma_{3}^{2}$ in $\mathrm{dBW}\left(\sigma_{2}^{2}=\sigma_{1}^{2}\right)$.

scheme against $E_{b}^{\text {AOW }}$. Overall, Fig. 4 shows that our scheme offers a better trade-off between consumed power and rate than the benchmark schemes and, in turn, this explains its significantly better $E_{b}$ performance.

Figure 5 complements the results of Fig. 4 by showing the extent of our scheme $E_{b}$ performance improvement in comparison with $E_{b}^{\mathrm{AOW}}$ (in Figs. 5 a) and c)) and $E_{b}^{\mathrm{TWB}}$ (in Figs. 5 b) and d)) in Settings $\mathbf{A}$ and $\mathbf{C}$ for a large range of $\sigma_{1}^{2}\left(\sigma_{2}^{2}=\sigma_{1}^{2}\right)$ and $\sigma_{3}^{2}$ values. The improvement is quantified in terms of the EE gain, which is defined as

$$
\Delta_{\mathrm{EE}}=100\left(1-E_{b}^{\star} / \chi\right) \%,
$$

where $\chi=E_{b}^{\mathrm{AOW}}$ or $\chi=E_{b}^{\mathrm{TWB}}$ in Fig 5. The results further confirm the very significant benefits of our scheme in comparison with the state-of-art in both the unconstrained (i.e. Figs. 5 a) and b)) and power constrained cases (i.e. Figs. 5 c) and d)). Our scheme achieves a EE gain of up to 40 and $60 \%$ against $E_{b}^{\mathrm{AOW}}$ and $E_{b}^{\mathrm{TWB}}$, respectively. It also show that the gain is very significant not only for specific $\sigma_{1}^{2}$ and $\sigma_{3}^{2}$ values (as in Fig. 4), but for a large range of these values.

Figure 6 compares the $E_{b}$ performance of our scheme against those of its lower and upper bounds, $E_{b}^{\mathbf{\nabla}}$ and $E_{b}^{\boldsymbol{\Delta}}$, respectively, in all the settings of Table II when $\sigma_{1}^{2}=\sigma_{2}^{2}=$ $\sigma_{3}^{2}=\sigma^{2}$. The results show a very good tightness between $E_{b}^{\star}$ and its bounds in most cases, except Settings $\mathbf{D}$ at high $\sigma^{2}$ values. Given that the complexity of finding $\mathbf{G}^{\star}$ in Algorithm 


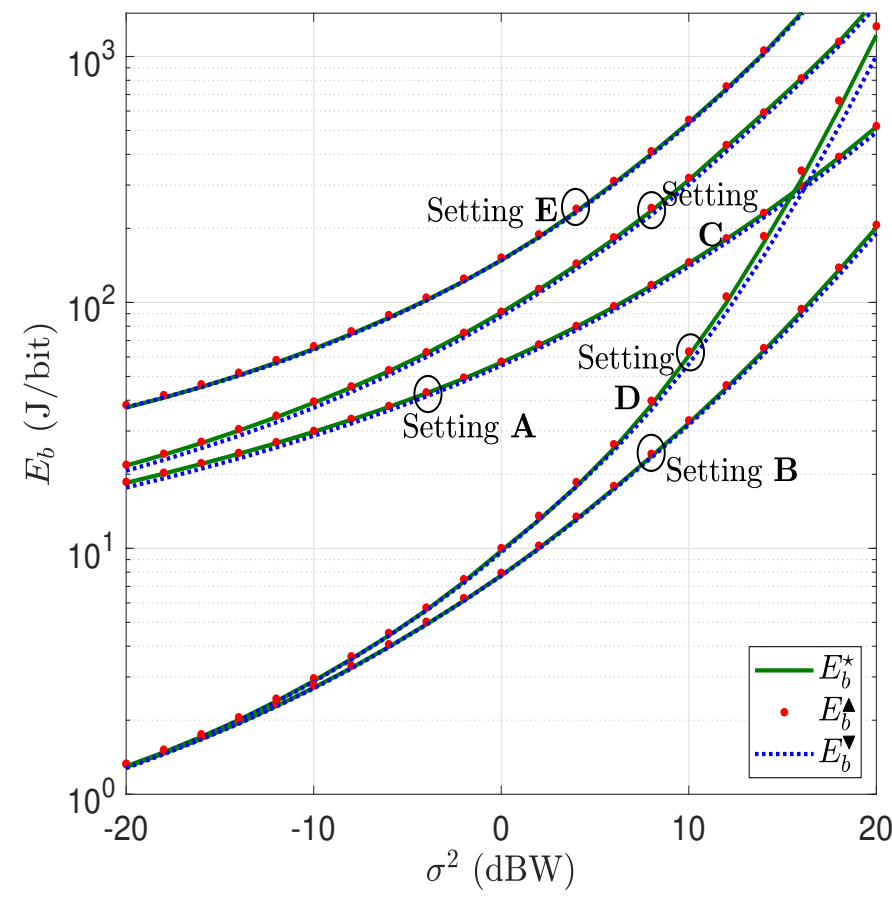

Fig. 6. Tightness of our lower and upper bounds of $E_{b}^{\star}$ as a function of $\sigma^{2}$ in $\mathrm{dBW}$ for five different settings.

3 is somehow linked to the gap between $E_{b}^{\boldsymbol{\nabla}}$ and $E_{b}^{\boldsymbol{\Delta}}$, i.e. $E_{b}^{\mathbf{\Delta}}-E_{b}^{\mathbf{\nabla}}$, this complexity should be acceptable in most of these cases. It can also be remarked that $E_{b}^{\star}$ is in general closer to its upper bound than its lower bound, and the gap between $E_{b}^{\boldsymbol{\Delta}}$ and $E_{b}^{\star}$ seems to always be relatively small. Hence, using Algorithm 1 in conjunction with a generic gradient/projected gradient search (instead of Algorithm 3) could be a reasonable sub-optimal alternative to achieve close to $E_{b}^{\star}$ performance but without the extra complexity of Algorithm 3. Meanwhile, it is clear that for the same number of antennas (i.e. settings $\mathbf{A}, \mathbf{B}$, C, D), lower values of the fixed power parameters (i.e. $P_{i}^{\text {CipA }}$ and $P_{i}^{\mathrm{Ci}}$ ) leads to lower energy consumption. Similarly, setting $\mathbf{E}$, which exhibits the highest/worst energy consumption, has the highest level of fixed power.

Figure 7 complements the results of Fig. 6 by depicting the EE gain of our scheme against its upper (in Figs. 7 a) and c)) and lower (in Figs. 7 b) and d)) bounds in Settings $\mathbf{A}$ and $\mathbf{C}$. The results in Figs. 7 a) and c) confirm the good tightness between $E_{b}^{\star}$ and its upper bound; they differ by up to only $3 \%$ over a large range of noise power values; this confirms that obtaining the RN precoding matrix through a generic gradient/projected gradient search method could be a reasonable sub-optimal alternative to Algorithm 3. Meanwhile, it can be remarked that for some settings, i.e. $\sigma_{1}^{2}=-\sigma_{3}^{2}$ with $\left|\sigma_{3}^{2}\right|>10 \mathrm{dBW}$ (left-upper and right lower corners of all the subplots of Fig. 7), both bounds closely match $E_{b}^{\star}$ results. This intuitively indicates that for this range of SNRs, the optimization problem in (17) is likely to be pseudo-convex (having a global optimum), since the upper bound is obtained via a gradient/projected gradient search method; a method that is only optimal for convex/pseudo-convex problem. This result also echoes the convergence analysis of Section V-B, since it indicates that if both $\sigma_{1}^{2} \ll \sigma_{3}^{2}$ and $\sigma_{2}^{2} \ll \sigma_{3}^{2}$ or $\sigma_{i}^{2} \gg \sigma_{3}^{2}$,

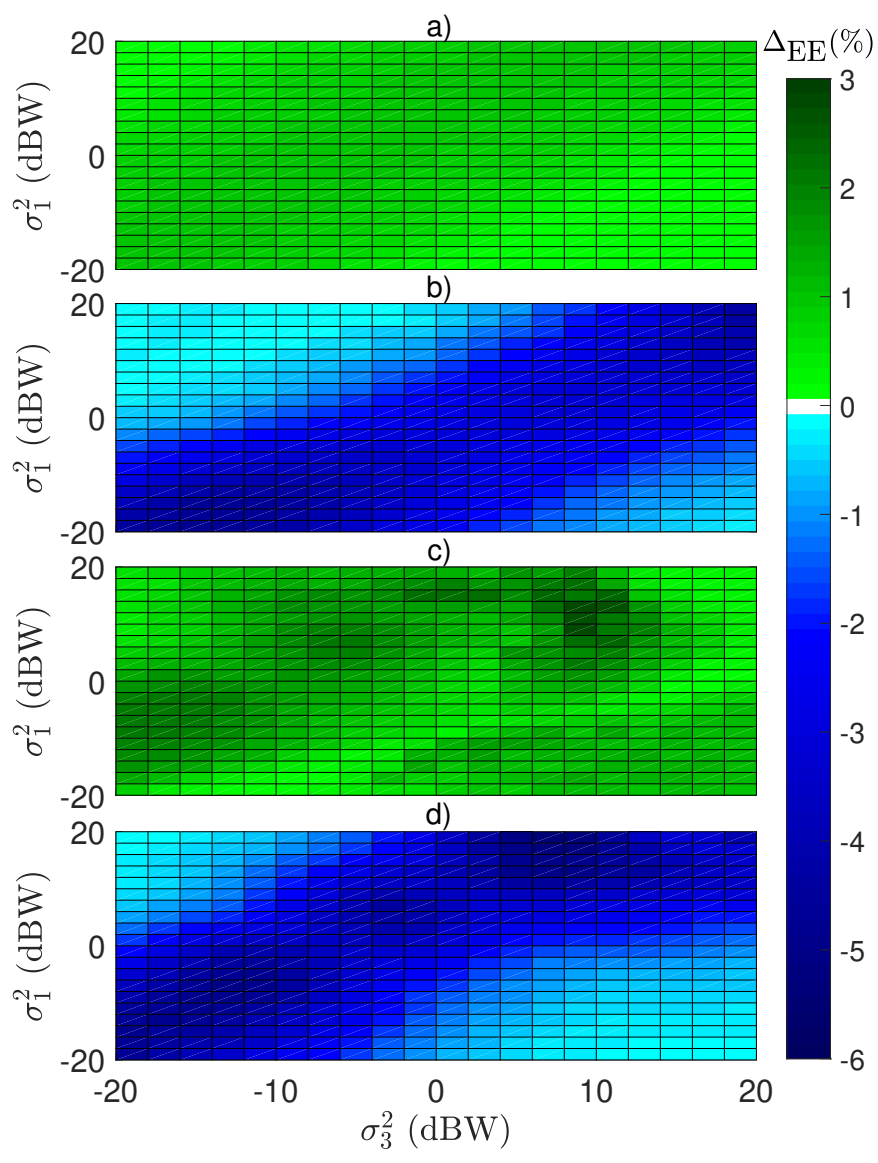

Fig. 7. EE gain comparison of our scheme against a) $E_{b}^{\mathbf{\Delta}}$ in Setting $\mathbf{A}$, b) $E_{b}^{\mathbf{\nabla}}$ in Setting $\mathbf{A}$, c) $E_{b}^{\mathbf{\Delta}}$ in Setting $\mathbf{C}$, and d) $E_{b}^{\mathbf{\nabla}}$ in Setting $\mathbf{C}$, as a function of $\sigma_{1}^{2}$ and $\sigma_{3}^{2}$ in $\operatorname{dBW}\left(\sigma_{2}^{2}=\sigma_{1}^{2}\right)$.

$i=1$ or 2 , then the optimization problem in (10) can converge to a global optimum.

Figure 8 depicts the variation of the energy consumption of our scheme as a function of the maximum transmit power, $P^{\max }(\mathrm{W})$, for different numbers of transmit antennas, $n$, at the three nodes. We assume that $\sigma_{1}^{2}=\sigma_{2}^{2}=\sigma_{3}^{2}=0 \mathrm{dBW}$ and use the power parameter values of Setting $\mathbf{A}$ for $\Delta_{i}$, $P_{i}^{\mathrm{CipA}}$ and $P_{i}^{\mathrm{Ci}}, i=1,2,3$. Note here that $P_{i}^{\max }=P^{\max }$ and $n_{i}=n, i=1,2,3$. The results show that, for any particular value of $n$, the energy consumption steadily decreases down to a minimum value as $P^{\max }$ increases. This is consistent with the discussion of Section III-B about the effects of constraints on optimization problem. Indeed, as $P^{\max }$ increases (i.e. the power constraints loosen), as the set of possible/feasible solutions increases and, hence, it is possible to find a lower value of $E_{b}$ in this set, up to the point where the minimum of the objective function (10a) itself is reached. Once the set of possible/feasible solution contains the minimum of (10a), then $P^{\max }$ has no more influence on the result, given that the minimum of (10a) acts a lower bound for (10). Meanwhile, Fig. 8 results also indicate that the energy consumption decreases as the number of antenna increases in the two-way MIMO-AF scenario, which is consistent with the results of [10] for the one-way MIMO-AF scenario. Even though increasing the number of transmit antennas increases the overall power consumption, as it is clearly indicated in (6), 


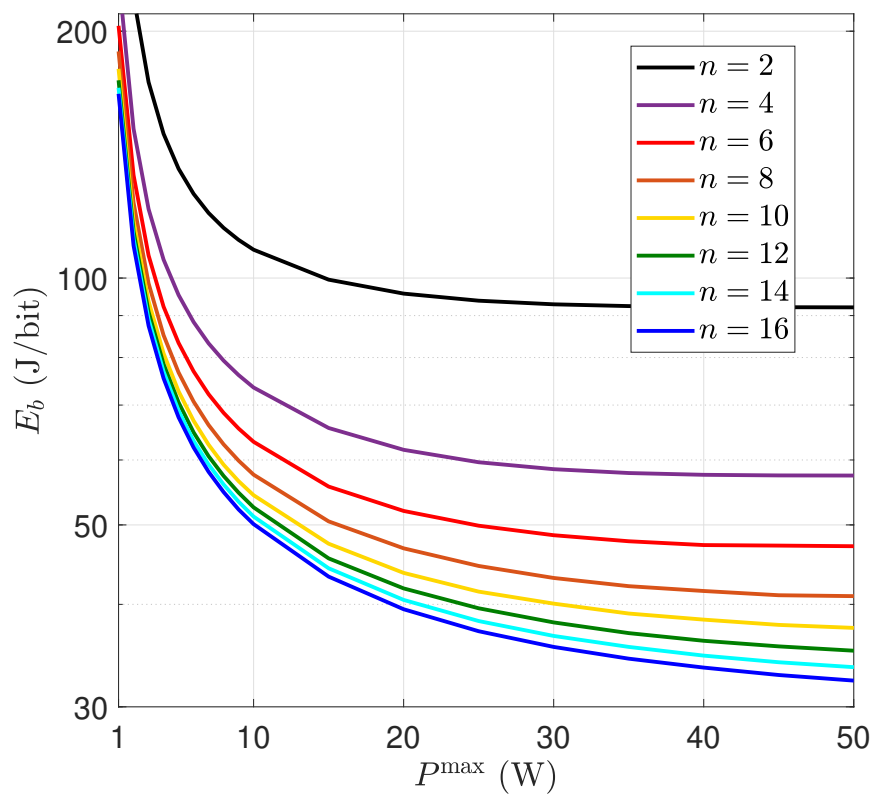

Fig. 8. EE performance of our scheme as a function of the maximum transmit power for different numbers of transmit antennas.

this increase in $n$ is more than compensated by the increase in rate brought by these extra antennas. However, it seems that this improvement in EE is logarithmic, i.e. the difference in energy consumption between having $n$ and $n+2$ antennas becomes smaller and smaller as $n$ increases. Note that similar result behaviours were observed for different values of $\sigma_{i}^{2}$.

\section{CONCLUSION}

In this paper, we have proposed a novel energy efficient precoding method for optimizing the EE/energy consumption of half-duplex two-way MIMO-AF relay systems. We have designed EE-optimal source and relay precoding matrices and the optimality of these matrices, when treated independently, has been formally proved by relying on pseudo-convexity analysis. An alternating optimization process (for which its convergence has been proved) has been used to jointly optimize these precoders. In addition, the EE-optimal source precoding matrices have been derived in closed-form and an optimal numerical approach has been designed for obtaining the relay precoding matrix. Performance evaluation has shown that our scheme can be globally optimal in some cases and can provide a very significant improvement in $\mathrm{EE}$ in comparison with relevant existing approaches; for instance, a EE performance improvement of up to $60 \%$ has been achieved compared to a reference EE-based two-way MIMO-AF beamforming scheme. In the future, we plan to generalize our scheme for any number of nodes, instead of three.

\section{APPENDIX}

\section{A. Derivation details: from Equation (3a) to (3b)}

Equation (3a) can be reformulated as

$$
W \sum_{i=1}^{2} \log _{2}\left|\mathbf{I}_{n_{\bar{i}}}+\mathbf{H}_{\bar{i} 3} \mathbf{G} \mathbf{H}_{3 i} \mathbf{R}_{i} \mathbf{R}_{i}^{\dagger} \mathbf{H}_{3 i}^{\dagger} \mathbf{G}^{\dagger} \mathbf{H}_{\bar{i} 3}^{\dagger} \mathbf{\Omega}_{i}^{-1}\right|,
$$

where $\boldsymbol{\Omega}_{i}=\sigma_{\bar{i}}^{2} \mathbf{I}_{n_{\bar{i}}}+\sigma_{3}^{2} \mathbf{H}_{\bar{i} 3} \mathbf{G G}^{\dagger} \mathbf{H}_{\bar{i} 3}^{\dagger}$. Then, by using the matrix determinant lemma, (32) can be re-expressed as

$$
\begin{aligned}
& W \sum_{i=1}^{2} \log _{2}\left|\mathbf{I}_{n_{i}}+\mathbf{R}_{i}^{\dagger} \mathbf{H}_{3 i}^{\dagger} \mathbf{G}^{\dagger} \mathbf{H}_{i 3}^{\dagger} \boldsymbol{\Omega}_{i}^{-1} \mathbf{H}_{\bar{i} 3} \mathbf{G H}_{3 i} \mathbf{R}_{i}\right| \\
\Leftrightarrow & W \sum_{i=1}^{2} \log _{2}\left|\mathbf{I}_{n_{i}}+\mathbf{R}_{i}^{\dagger} \dot{\Psi}_{i}(\mathbf{G}) \mathbf{R}_{i}\right| .
\end{aligned}
$$

Moreover, knowing that for any matrices $\mathbf{A}$ and $\mathbf{B}$ of dimensions $n_{\mathrm{A}} \times n_{\mathrm{A}}$ and $n_{\mathrm{B}} \times n_{\mathrm{B}}$, correspondingly,

$$
|\mathbf{A}||\mathbf{B}|=\left|\begin{array}{cc}
\mathbf{A} & \mathbf{0} \\
\mathbf{0} & \mathbf{B}
\end{array}\right| \Rightarrow \log _{2}|\mathbf{A}|+\log _{2}|\mathbf{B}|=\log _{2}\left|\begin{array}{cc}
\mathbf{A} & \mathbf{0} \\
\mathbf{0} & \mathbf{B}
\end{array}\right|,
$$

it implies that (3b) is equivalent to (33). Hence, (3b) $\Leftrightarrow$ (33) $\Leftrightarrow(32) \Leftrightarrow(3 a)$.

\section{B. Proof of Proposition 3}

Proof: Given the power constraints in (11b), it implies that $P_{i}(\mathbf{Y})$ in (11a) is defined as in (12b), when $P_{i}(\mathbf{Y})<$ $P_{i}^{\max }$; whereas $P_{i}(\mathbf{Y})=P_{i}^{\max }$ when $P_{i}(\mathbf{Y}) \geq P_{i}^{\max }$. In other words, $P_{i}(\mathbf{Y})$ is either dependent or independent of $\mathbf{Y}$ when $P_{i}(\mathbf{Y})<$ or $\geq P_{i}^{\max }$, respectively. Consequently, the Lagrangian function associated with the optimization problem in (11) can be formulated as

$$
\begin{aligned}
\mathcal{L}(\boldsymbol{\mu}, \mathbf{Y}) & =\frac{P_{c}+\sum_{i=1}^{3} \Delta_{i}\left(\delta_{i} P_{i}(\mathbf{Y})+\left(1-\delta_{i}\right) P_{i}^{\max }\right)}{R_{\Sigma}(\mathbf{Y})} \\
& +\sum_{i=1}^{3} \mu_{i}\left(1-\delta_{i}\right)\left(P_{i}(\mathbf{Y})-P_{i}^{\max }\right),
\end{aligned}
$$

where $\boldsymbol{\mu}=\left[\mu_{1}, \mu_{2}, \mu_{3}\right], \mu_{i}$ is a Lagrange multiplier, and

$$
\delta_{i}= \begin{cases}1, & \text { if } P_{i}(\mathbf{Y}) \leq P_{i}^{\max } \\ 0, & \text { else. }\end{cases}
$$

The optimization problem in (11) being pseudo-convex, the global optimum of $\mathcal{L}(\boldsymbol{\mu}, \mathbf{Y})$ occurs at a stationary point (see theorem 1.36 of [37]), such that

$$
\nabla_{\mathbf{Y}} \mathcal{L}\left(\boldsymbol{\mu}=\boldsymbol{\mu}^{\star}, \mathbf{Y}=\mathbf{Y}^{\star}\right)=\mathbf{0},
$$

where $\boldsymbol{\mu}^{\star}=\boldsymbol{\mu} R_{\Sigma}\left(\mathbf{Y}^{\star}\right)$, and based on (35), $\nabla_{\mathbf{Y}} \mathcal{L}(\boldsymbol{\mu}, \mathbf{Y})=$

$$
\begin{aligned}
& \frac{R_{\Sigma}(\mathbf{Y}) \sum_{i=1}^{3} \Delta_{i} \delta_{i} \nabla_{\mathbf{Y}} P_{i}(\mathbf{Y})-P_{\Sigma}(\mathbf{Y}) \nabla_{\mathbf{Y}} R_{\Sigma}(\mathbf{Y})}{R_{\Sigma}(\mathbf{Y})^{2}} \\
& +\sum_{i=1}^{3} \mu_{i}\left(1-\delta_{i}\right) \nabla_{\mathbf{Y}} P_{i}(\mathbf{Y}) .
\end{aligned}
$$

Moreover $\nabla_{\mathbf{Y}} P_{i}(\mathbf{Y})=\boldsymbol{\Psi}_{i}$ and $\nabla_{\mathbf{Y}} R_{\Sigma}(\mathbf{Y})=$ $\frac{W}{\ln (2)}\left(\mathbf{I}_{n_{0}}+\mathbf{Y}\right)^{-1}$ based on (12); hence, equation (37) is equivalent to

$$
\frac{\sum_{i=1}^{3}\left(\delta_{i}^{\star} \Delta_{i}+\left(1-\delta_{i}^{\star}\right) \mu_{i}^{\star}\right) \mathbf{\Psi}_{i}-\frac{W E_{b}^{\star}}{\ln (2)}\left(\mathbf{I}_{n_{0}}+\mathbf{Y}^{\star}\right)^{-1}}{R_{\Sigma}\left(\mathbf{Y}^{\star}\right)}=\mathbf{0},
$$

where $E_{b}^{\star}=E_{b}\left(\mathbf{Y}^{\star}\right)=\frac{P_{\Sigma}\left(\mathbf{Y}^{\star}\right)}{R_{\Sigma}\left(\mathbf{Y}^{\star}\right)}$ and $\delta_{i}^{\star}$ is defined as in (36) but for $\mathbf{Y}=\mathbf{Y}^{\star}$. Let $\alpha_{i}=\delta_{i}^{\star} \Delta_{i}+\left(1-\delta_{i}^{\star}\right) \mu_{i}^{\star}, i=1,2,3$, 
then (39) simplifies as

$$
\begin{gathered}
\sum_{i=1}^{3} \alpha_{i} \boldsymbol{\Psi}_{i}-\frac{W E_{b}^{\star}}{\ln (2)}\left(\mathbf{I}_{n_{0}}+\mathbf{Y}^{\star}\right)^{-1}=\mathbf{0}, \\
\Leftrightarrow \mathbf{Y}^{\star}=\frac{W E_{b}^{\star}}{\ln (2)}\left(\sum_{i=1}^{3} \alpha_{i} \boldsymbol{\Psi}_{i}\right)^{-1}-\mathbf{I}_{n_{0}} .
\end{gathered}
$$

Finally, given that $\mathbf{Y}=\dot{\boldsymbol{\Psi}}^{\frac{1}{2}} \mathbf{R} \mathbf{R}^{\dagger} \dot{\boldsymbol{\Psi}}^{\frac{1}{2}}$, then $\mathbf{Y}^{\star}=$ $\dot{\boldsymbol{\Psi}}^{\frac{1}{2}} \mathbf{R}^{\star}\left(\mathbf{R}^{\star}\right)^{\dagger} \dot{\boldsymbol{\Psi}}^{\frac{1}{2}} \Leftrightarrow \mathbf{R}^{\star}\left(\mathbf{R}^{\star}\right)^{\dagger}=\dot{\boldsymbol{\Psi}}^{-\frac{1}{2}} \mathbf{Y}^{\star} \dot{\boldsymbol{\Psi}}^{-\frac{1}{2}}$, which leads to (15). It should also be noted that since $\sum_{i=1}^{3} \alpha_{i} \boldsymbol{\Psi}_{i}$ in (40b) is a block diagonal matrix, then its inverse is also block diagonal, such that $\mathbf{Y}^{\star}$ is a block diagonal matrix that meets the requirement of the optimization problem in (11).

\section{Proof of Proposition 4}

Proof: On the one hand, according to (3a),

$$
R_{\Sigma}(\mathbf{G})=W \sum_{i=1}^{2} \log _{2}\left|\mathbf{I}_{n_{\bar{i}}}+\frac{\frac{\sigma_{3}^{2}}{\sigma_{\bar{i}}^{2}} \mathbf{H}_{\bar{i} 3} \mathbf{G}_{i} \dot{\boldsymbol{\Upsilon}}_{i} \mathbf{G}_{i}^{\dagger} \mathbf{H}_{\bar{i} 3}^{\dagger}}{\mathbf{I}_{n_{\bar{i}}}+\frac{\sigma_{3}^{2}}{\sigma_{\bar{i}}^{2}} \mathbf{H}_{\bar{i} 3} \mathbf{G}_{i} \mathbf{G}_{i}^{\dagger} \mathbf{H}_{\bar{i} 3}^{\dagger}}\right|,
$$

when $\mathbf{G}_{1}=\mathbf{G}_{2}=\mathbf{G}$. Let $\overline{\mathbf{G}}=\left[\mathbf{G}_{1} \mathbf{0} ; \mathbf{0} \mathbf{G}_{2}\right] \in \mathbb{C}^{2 n_{3} \times 2 n_{3}}$ be a block diagonal matrix, and $\mathbf{H}_{3}$ as well as $\boldsymbol{\Upsilon}$ be defined as in (21), it then implies with (34) (i.e. property of a block matrix determinant) that $R_{\Sigma}(\mathbf{G})$ in (41), as well as in (3a), is equivalent to $R_{\Sigma}(\overline{\mathbf{G}})$ in (20a) for $\mathbf{G}_{1}=\mathbf{G}_{2}=\mathbf{G}$ in (18). On the other hand, according to $(7 b)$,

$$
\begin{aligned}
P_{3}(\mathbf{G}) & =\sigma_{3}^{2} \operatorname{tr}\left\{\mathbf{G}\left(\mathbf{I}_{n_{3}}+\sum_{i=1}^{2} \dot{\boldsymbol{\Upsilon}}_{i}\right) \mathbf{G}^{\dagger}\right\}, \\
& =\sigma_{3}^{2} \sum_{i=1}^{2} \operatorname{tr}\left\{\mathbf{G}_{i}\left(\frac{\mathbf{I}_{n_{3}}}{2}+\dot{\boldsymbol{\Upsilon}}_{i}\right) \mathbf{G}_{i}^{\dagger}\right\},
\end{aligned}
$$

when $\mathbf{G}_{1}=\mathbf{G}_{2}=\mathbf{G}$. Knowing that for any matrices $\mathbf{A}$ and $\mathbf{B}$ of dimensions $n_{\mathrm{A}} \times n_{\mathrm{A}}$ and $n_{\mathrm{B}} \times n_{\mathrm{B}}$, respectively,

$$
\operatorname{tr}\{\mathbf{A}\}+\operatorname{tr}\{\mathbf{B}\}=\operatorname{tr}\left\{\left[\begin{array}{cc}
\mathbf{A} & \mathbf{0} \\
\mathbf{0} & \mathbf{B}
\end{array}\right]\right\}
$$

and based on the definition of $\Upsilon$ in (21), it can be concluded that $P_{3}(\mathbf{G})$ in (42), as well as in $(7 \mathrm{~b})$, is equivalent to $P_{3}(\overline{\mathbf{G}})$ in (20b) for $\mathbf{G}_{1}=\mathbf{G}_{2}=\mathbf{G}$ in (18). Hence, based on (41) and (42), the optimization problem in (19) is equivalent to the problem in (17) for $\mathbf{G}_{1}=\mathbf{G}_{2}=\mathbf{G}$ in (18).

\section{Proof of the closed-form in (25)}

Proof: Similar to Section B of the Appendix, $P_{3}(\mathbf{g})$ is defined as in (23b) when $P_{3}(\mathbf{g})<P_{3}^{\max }$ or $P_{3}(\mathrm{~g})=P_{3}^{\max }$ when $P_{3}(\mathbf{g}) \geq P_{3}^{\max }$, such that $P_{3}(\mathbf{g})$ is either dependent or independent of $\mathbf{g}$. Consequently, the Lagrangian function associated with the optimization problem in (23) can be formulated as

$$
\begin{aligned}
\mathcal{L}\left(\mu_{3}, \mathbf{g}\right) & =\frac{P_{c}^{\prime}+\Delta_{3}\left(\delta_{3} P_{3}(\mathbf{g})+\left(1-\delta_{3}\right) P_{3}^{\max }\right)}{R_{\Sigma}(\mathbf{g})} \\
& +\mu_{3}\left(1-\delta_{i}\right)\left(P_{3}(\mathbf{g})-P_{3}^{\max }\right)
\end{aligned}
$$

where $\mu_{3}$, is a Lagrange multiplier, and $\delta_{3}=1$ if $P_{3}(\mathbf{g}) \leq$ $P_{3}^{\max }$ or $\delta_{3}=0$ else. Given that (23) is pseudo-convex (i.e.
(23) is a ratio of linear to a strictly concave function; see a proof in [9]), the global optimum of $\mathcal{L}\left(\mu_{3}, \mathbf{g}\right)$ occurs at a stationary point (see theorem 1.36 of [37]), such that

$$
\nabla_{\mathbf{g}} \mathcal{L}\left(\mu_{3}=\mu_{3}^{\mathbf{\nabla}}, \mathbf{g}=\mathbf{g}^{\mathbf{\nabla}}\right)=\mathbf{0},
$$

where $\nabla_{\mathbf{g}} \mathcal{L}\left(\mu_{3}, \mathbf{g}\right)=\left[\frac{\partial \mathcal{L}\left(\mu_{3}, \mathbf{g}\right)}{\partial g_{1}}, \frac{\partial \mathcal{L}\left(\mu_{3}, \mathbf{g}\right)}{\partial g_{2}}, \ldots, \frac{\partial \mathcal{L}\left(\mu_{3}, \mathbf{g}\right)}{\partial g_{2 n_{3}}}\right]$ and $\mu_{3}^{\boldsymbol{\nabla}}=\mu_{3} R_{\Sigma}\left(\mathbf{g}^{\mathbf{\nabla}}\right)$. Then, according to (43),

$\frac{\partial \mathcal{L}\left(\mu_{3}, \mathbf{g}\right)}{\partial g_{j}}=\frac{\Delta_{3} \delta_{3} \frac{P_{3}(\mathbf{g})}{\partial g_{j}}}{R_{\Sigma}(\mathbf{g})}-\frac{P_{\Sigma}(\mathbf{g}) \frac{R_{\Sigma}(\mathbf{g})}{\partial g_{j}}}{R_{\Sigma}(\mathbf{g})^{2}}+\mu_{3}\left(1-\delta_{3}\right) \frac{P_{3}(\mathbf{g})}{\partial g_{j}}$,

$j=1,2, \ldots, 2 n_{3}$, with $\frac{P_{3}(\mathbf{g})}{\partial g_{j}}=\sigma_{3}^{2}\left(1 / 2+v_{j}\right)$ and $\frac{R_{\Sigma}(\mathbf{g})}{\partial g_{j}}=$ $\frac{W}{\ln (2)}\left(\frac{h_{j}\left(1+v_{j}\right)}{1+g_{j} h_{j}\left(1+v_{j}\right)}+\frac{h_{j}}{1+g_{j} h_{j}}\right)$ based on (23b) and (24), correspondingly. Let $E_{b}^{\mathbf{\nabla}}=E_{b}\left(\mathbf{g}^{\mathbf{\nabla}}\right)=\frac{P_{\Sigma}\left(\mathbf{g}^{\mathbf{\nabla}}\right)}{R_{\Sigma}\left(\mathbf{g}^{\mathbf{v}}\right)}, \delta_{3}^{\mathbf{\nabla}}=1$ if $P_{3}\left(\mathbf{g}^{\mathbf{\nabla}}\right) \leq P_{3}^{\max }$ or $\delta_{3}^{\boldsymbol{\nabla}}=0$ else, and $\alpha_{3}=\delta_{3}^{\mathbf{\nabla}} \Delta_{3}+\left(1-\delta_{3}^{\mathbf{\nabla}}\right) \mu_{3}^{\boldsymbol{\nabla}}$, then, according to (45), equation (44) is equivalent to

$$
\begin{array}{r}
\alpha_{3} \sigma_{3}^{2}\left(1 / 2+v_{j}\right)=\frac{W E_{b}^{\mathbf{\nabla}}}{\ln (2)}\left(\frac{h_{j}\left(1+v_{j}\right)}{1+g_{j} h_{j}\left(1+v_{j}\right)}-\frac{h_{j}}{1+g_{j} h_{j}}\right), \\
\Leftrightarrow g_{j}^{2} h_{j}\left(1+v_{j}\right)+g_{j}\left(2+v_{j}\right)+\frac{1}{h_{j}}-\frac{2 W E_{b}^{\mathbf{\nabla}} v_{j}}{\ln (2) \alpha_{3} \sigma_{3}^{2}\left(1+2 v_{j}\right)}=0 .
\end{array}
$$

Finally, equation (25) is the largest root (out of two) of the quadratic equation in (46b).

\section{E. Proof of Equation 27}

Proof: Any extrema of a differentiable function can only occur at stationary points according to (p.194 of [38]). Hence, the global minimum of $E_{b}, E_{b}^{\star}=E_{b}\left(\mathbf{G}^{\star}\right)$, (if it exists) must occur at a stationary point such that $\nabla_{\mathbf{G}} E_{b}\left(\mathbf{G}=\mathbf{G}^{\star}\right)=\mathbf{0}$, or equivalently,

$$
\begin{aligned}
& \frac{1}{R_{\Sigma}\left(\mathbf{G}^{\star}\right)}\left[\nabla_{\mathbf{G}} P_{\Sigma}\left(\mathbf{G}^{\star}\right)-E_{b}\left(\mathbf{G}^{\star}\right) \nabla_{\mathbf{G}} R_{\Sigma}\left(\mathbf{G}^{\star}\right)\right]=\mathbf{0} . \\
\Leftrightarrow & \nabla_{\mathbf{G}} P_{\Sigma}\left(\mathbf{G}^{\star}\right)-E_{b}\left(\mathbf{G}^{\star}\right) \nabla_{\mathbf{G}} R_{\Sigma}\left(\mathbf{G}^{\star}\right)=\mathbf{0}, \\
\Leftrightarrow & \nabla_{\mathbf{G}} P_{\Sigma}\left(\mathbf{G}^{\star}\right)-\left(E_{b}^{\mathbf{\nabla}}+\varepsilon^{\star}\right) \nabla_{\mathbf{G}} R_{\Sigma}\left(\mathbf{G}^{\star}\right)=\mathbf{0},
\end{aligned}
$$

which leads to (27).

\section{ACKNOWLEDGMENT}

The first author wishes to thank his beloved wife for her support.

\section{REFERENCES}

[1] H. Zhang et al., "Energy efficiency in communications," IEEE Commun. Mag., vol. 48, no. 11, pp. 48-79, Nov. 2010.

[2] L. M. Correia et al., "Challenges and enabling technologies for energy aware mobile radio networks," IEEE Commun. Mag., vol. 48, no. 11, pp. 66-72, Nov. 2010.

[3] G. Li et al., "Energy efficient wireless communications: tutorial, survey, and open issues," IEEE Wireless Commun., vol. 18, no. 6, pp. 28-35, Dec. 2011.

[4] International Telecommunication Union (ITU), "IMT Vision - Framework and overall objectives of the future development of IMT for 2020 and beyond," ITU, Geneva, Switzerland, ITU-R Recommendations M.2083-0, Sep. 2015.

[5] A. Sendonaris, E. Erkip, and B. Aazhang, "User cooperation diversity part I \& II- system description/implementation aspects and performance analysis," IEEE Trans. Commun., vol. 51, no. 11, pp. 1927-1948, Nov. 2003. 
[6] A. Nosratinia, T. E. Hunter, and A. Hedayat, "Cooperative communication in wireless networks," IEEE Commun. Mag., vol. 42, no. 10, pp. 74-80, Oct. 2004.

[7] J. N. Laneman, D. N. C. Tse, and G. W. Wornell, "Cooperative diversity in wireless networks: efficient protocols and outage behavior," IEEE Trans. Inf. Theory, vol. 50, no. 12, pp. 3062-3080, Dec. 2004.

[8] J. Vidal et al. (2008-2009) Reconfigurable OFDMA-based Cooperative NetworKs Enabled by Agile SpecTrum Use (ROCKET). Universitat Politecnica de Catalunya and others. Barcelona, Spain. [Online]. Available: http://cordis.europa.eu/project/rcn/85296_en.html

[9] A. Zappone, P. Cao, and E. A. Jorswieck, "Energy efficiency optimization in relay-assisted MIMO systems with perfect and statistical CSI," IEEE Trans. Signal Process., vol. 62, no. 2, pp. 443-457, Jan. 2014.

[10] F. Héliot, "Low-complexity energy-efficient joint resource allocation for two-hop MIMO-AF systems," IEEE Trans. Wireless Commun., vol. 13, no. 6, pp. 3088-3099, Jun. 2014.

[11] RAN WG1 (Radio layer 1), "Evolved Universal Terrestrial Radio Access (E-UTRA); Physical layer for relaying operation (Release 10-14)," The 3rd Generation Partnership Project (3GPP), Technical Specifications 36.216, 2010 onwards. [Online]. Available: http: //www.3gpp.org/dynareport/36216.htm

[12] R. I. Ansari et al., "5G D2D networks: techniques, challenges, and future prospects," IEEE Syst. J., vol. 12, no. 4, pp. 3970-3984, Dec. 2018.

[13] L. Sanguinetti, A. A. D'Amico, and Y. Rong, "A tutorial on the optimization of amplify-and-forward MIMO relay systems," IEEE J. Sel. Areas Commun., vol. 30, no. 8, pp. 1331-1346, Sep. 2012.

[14] O. Muñoz-Medina, J. Vidal, and A. Agustín, "Linear transceiver design in nonregenerative relays with channel state information," IEEE Trans. Signal Process., vol. 55, no. 6, pp. 2593-2604, Jun. 2007.

[15] K.-J. Lee, K. Lee, H. Sung, and I. Lee, "Sum-rate maximization for two-way MIMO amplify-and-forward relaying systems," in Proc. IEEE VTC-Spring, Barcelona, Spain, Apr. 2009.

[16] S. Xu and Y. Hua, "Optimal design of spatial source-and-relay matrices for a non-regenerative two-way MIMO relay system," IEEE Trans. Wireless Commun., vol. 10, no. 5, pp. 1645-1655, Nov. 2011.

[17] R. Wang and M. Tao, "Joint source and relay precoding designs for MIMO two-way relaying based on MSE criterion," IEEE Trans. Signal Process., vol. 60, no. 3, pp. 1352-1365, Mar. 2012.

[18] Y. Rong, X. Tang, and Y. Hua, "Joint source and relay optimization for two-way Linear non-regenerative MIMO relay communications," IEEE Trans. Signal Process., vol. 60, no. 12, pp. 6533-6546, Dec. 2012.

[19] J. Zhang and M. Haardt, "Energy efficient two-way non-regenerative relaying for relays with multiple antennas," IEEE Signal Process. Lett., vol. 22, no. 8, pp. 1079-1083, Aug. 2015.

[20] F. Héliot and R. Tafazolli, "Optimal energy-efficient joint resource allocation for multi-hop MIMO-AF systems," IEEE Trans. Commun., vol. 64, no. 9, pp. 3655-3668, Sep. 2016.

[21] Z. Wang, L. Li, H. Tian, and A. Paulraj, "User-centric precoding designs for the non-regenerative MIMO two-way relay systems," IEEE Commun. Lett., vol. 20, no. 10, pp. 1935-1938, Oct. 2016.

[22] Z. Sheng, H. D. Tuan, T. Q. Duong, and H. V. Poor, "Joint power allocation and beamforming for energy-efficient two-way multi-relay communications," IEEE Trans. Wireless Commun., vol. 16, no. 10, pp. 6660-6671, Oct. 2017.

[23] F. Héliot and R. Tafazolli, "Optimal energy-efficient source and relay precoder design for cooperative MIMO-AF systems," IEEE Trans. Signal Process., vol. 66, no. 3, pp. 573-588, Feb. 2018.

[24] R. Hunger et al., "Alternating optimization for MMSE broadcast precoding," in Proc. IEEE ICASSP, Toulouse, France, May 2006, pp. 757-760.

[25] F. Héliot and R. Tafazolli, "Energy-efficient sources and relay precoding design for two-way two-hop MIMO-AF systems," in Proc. EuCNC, Ljubljana, Slovenia, Jun. 2018.

[26] C. Xing et al., "A general robust linear transceiver design for multihop amplify-and-forward MIMO relaying systems," IEEE Trans. Signal Process., vol. 61, no. 5, pp. 1196-1209, Mar. 2013.

[27] G. Auer et al., "How much energy is needed to run a wireless network ?" IEEE Wireless Commun., vol. 18, no. 5, pp. 40-49, Oct. 2011.

[28] Y. Qi, F. Héliot, and M. A. Imran, "Green relay techniques in cellular systems," in Green communications and networking, F. R. Yu, X. Zhang, and V. C. Leung, Eds. Boca Raton, FL: CRC press, Dec. 2012, ch. 3.

[29] G. Miao, N. Himayat, and G. Y. Li, "Energy-efficient link adaptation in frequency-selective channels," IEEE Trans. Commun., vol. 58, no. 2 , pp. 545-554, Feb. 2010.

[30] F. Héliot, M. A. Imran, and R. Tafazolli, "On the energy efficiencyspectral efficiency trade-off over the MIMO Rayleigh fading channel," IEEE Trans. Commun., vol. 60, no. 5, pp. 1345-1356, May 2012.
[31] — - "Joint source and relay energy-efficient resource allocation for two-hop MIMO-AF systems," in Proc. IEEE ICC, Sidney, Australia, Jun. 2014.

[32] S. Verdu, "Spectral efficiency in the wideband regime," IEEE Trans. Inf. Theory, vol. 48, no. 6, pp. 1319-1343, Jun. 2002.

[33] R. W. Freund and F. Jarre, "An interior-point method for fractional programs with convex constraints," Math. Program., vol. 67, no. 1, pp. 407-440, Oct. 1994.

[34] S. Boyd and L. Vandenberghe, Convex optimization. Cambridge, UK: Cambridge Univ. Press, Mar. 2004.

[35] E. W. Weisstein, "Newton's method." MathWorld-A Wolfram Web Resource. [Online]. Available: http://mathworld.wolfram.com/ NewtonsMethod.html

[36] K. B. Petersen and M. S. Pedersen, "The matrix cookbook," nov 2012. [Online]. Available: http://www2.imm.dtu.dk/pubdb/p.php?3274

[37] Q. H. Ansari, C. S. Lalitha, and M. Mehta, Generalized Convexity, Nonsmooth Variational Inequalities, and Nonsmooth Optimization. NewYork, Chapman and Hall/CRC, Jul. 2013.

[38] M. Golden, Mathematical methods for neural network analysis and design. Cambridge, MA: MIT Press, Dec. 1996.

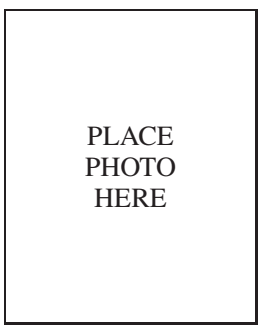

Fabien Héliot (S'05-M'07) received the MSc degree in Telecommunications from the Institut Supérieur de l'Electronique et du Numérique (ISEN), Toulon, France, and the PhD degree in Mobile Telecommunications from King's College London, in 2002 and 2006, respectively. He is currently a Lecturer at the Institute for Communication Systems (ICS) of the University of Surrey, formerly known as CCSR. He has been actively involved in European Commission (EC) funded projects such as FIREWORKS, ROCKET, SMART-Net, LEXNET projects and in the award-winning EARTH project. He is currently involved in the UK-funded 5 GIC and EU-funded 5GRFEX projects. His research interests include energy efficiency, EM exposure reduction, cooperative communication, MIMO, and radio resource management. He received an Exemplary Reviewer Award from IEEE COMMUNICATIONS LETTERS in 2011.

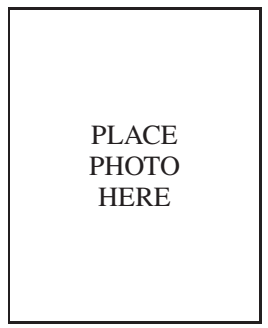

Rahim Tafazolli (SM'09) is a professor and the Director of the Institute for Communication Systems (ICS) and 5G Innovation Centre (5GIC), University of Surrey, United Kingdom. He has been active in research for more than 25 years and has authored and co-authored more than 800 papers in refereed international journals and conferences. Professor Tafazolli is a consultant to many mobile companies, has lectured, chaired, and been invited as keynote speaker to a number of IET and IEEE workshops and conferences. He has been Technical Advisor to many mobile companies, all in the field of mobile communications. He is the Founder and past Chairman of IET International Conference on 3rd Generation Mobile Communications. He is a Fellow of the IET and WWRF (Wireless World Research Forum). He is Chairman of EU Expert Group on Net!Works Technology Platform. 